\title{
Mapping Robust Genetic Variants Associated with Exercise Responses
}

\author{
Authors \\ Javier Alvarez-Romero', Sarah Voisin'1, Nir Eynon', 2, Danielle Hiam'D
}

\author{
Affiliations \\ 1 Institute for Health and Sport, Victoria University, \\ Melbourne, Australia \\ 2 MCRI, Murdoch Childrens Research Institute, Parkville, \\ Australia
}

Key words

trainability, aerobic exercise, exercise genetics, resistance exercise, SNPs, genetic variants

accepted $\quad 01.06 .2020$

published online 22.07 .2020

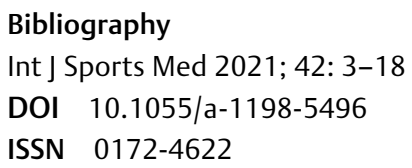

(C) 2020. Thieme. All rights reserved. Georg Thieme Verlag KG, Rüdigerstraße 14, 70469 Stuttgart, Germany

\author{
Correspondence \\ Dr. Nir Eynon \\ Institue of Sports Exercise and Active Living, Victoria \\ University \\ 8001 Melbourne, \\ Australia \\ Tel.: +61 399195615, Fax : +61399195615 \\ Nir.Eynon@vu.edu.au
}

\begin{abstract}
This review summarised robust and consistent genetic variants associated with aerobic-related and resistance-related phenotypes. In total we highlight 12 SNPs and 7 SNPs that are robustly associated with variance in aerobic-related and resistance-related phenotypes respectively. To date, there is very little literature ascribed to understanding the interplay between genes and environmental factors and the development of physiological traits. We discuss future directions, including large-scale exercise studies to elucidate the functional relevance of the discovered genomic markers. This approach will allow more rigour and reproducible research in the field of exercise genomics.
\end{abstract}

\section{Introduction}

Both aerobic and strength exercise training lower the incidence of many chronic diseases via a number of mechanisms, including increased skeletal muscle mitochondrial function [1], modulation of the sympathetic nervous and immune systems, and optimization of the neuroendocrine system [2]. These mechanisms act as buffers against chronic diseases, minimizing inflammatory state, and enhancing neuroplasticity and growth factor expression [3]. However, large inter-individual differences exist in the physiological responses to any given exercise training (also called "trainability") $[4,5]$, and recently new statistical methods have been developed to properly isolate individual responses from random error [6]. Large trainability has been observed in many physical fitness parameters [7], including maximal oxygen uptake $\left(\mathrm{VO}_{2} \mathrm{max}\right)[8,9]$, resting heart rate [9], exercise heart rate [9], aerobic threshold [10], anaerobic threshold [9], resting muscle glycogen content, muscle enzyme activity [11], as well as muscle mass and strength [12,13].

The heritable component of trainability is large, with genetics explaining $47 \%$ of the variance in $\mathrm{VO}_{2}$ peak trainability, and around $52 \%$ in resistance variability [14]. The contribution of familial factors (genetics and environment) to trainability was demonstrated in the seminal HERITAGE family study [15]. This study indicated that $\mathrm{VO}_{2}$ max was more variable between families than within families at baseline [16], and in response to exercise training [17], thus suggesting that DNA sequence variations could modulate exercise responses $[4,18]$. Pinpointing the responsible gene variants could illuminate the fundamental mechanisms driving this heterogeneity in response to exercise training [18].

The genetic contribution to trainability has been investigated by two different approaches: candidate genes and genome-wide association (GWAS) study. The GWAS approach involves scanning 
several hundred thousand (currently up to 5 million) DNA markers across the human genome to find genetic variations associated with a particular trait. One of the advantages of the GWAS approach is that it is unbiased and hypothesis-free. In contrast, candidate gene studies require knowledge of the trait of interest and is particularly useful to validate the functional impact of gene loci such as those identified by GWAS [19]. GWAS have demonstrated that trainability is polygenic (i. e., influenced by many genetic variants), and that people harbouring the same genotypes in specific gene variants respond more similarly to exercise training than people harbouring different genotypes [20-23]. These variants may modulate gene expression that is essential to the molecular adaptation to exercise training, since molecular processes mediate metabolism, angiogenesis, cardiac and skeletal myofibre hypertrophy, and other processes that lead to better fitness [24].

While many SNPs have been associated with exercise response and trainability. The vast majority of the genes previously identified have not been replicated [25]. Replication in an independent cohort is important as it increases the likelihood that results are true and reduces the number of false positives [26, 27]. In this review we summarised SNPs associated with both resistance and aerobic trainability and have been replicated in two independent cohorts. In addition, we have screened these SNPs with the goal of identifying SNPs at trainability-associated loci that may have functional relevance. Further, we discussed future directions of performing large-scale exercise studies to elucidate the functional relevance of the discovered genomic markers. This approach will allow more rigour and reproducible research in the field of exercise genomics.

\section{Materials and Methods}

To provide a robust and comprehensive narrative review, a semistructured search was performed (July 2019) to identify all studies relating to genetic variants and exercise trainability. Three electronic databases (PUBMED, MEDLINE and SCOPUS) were used to identify relevant articles using the following keywords "genes", "genome”, "exercise”, "physical activity”, "aerobic capacity”, “resistance", "strength”, "power”. We excluded studies where the sole focus was on populations with a diagnosed medical condition such type 2 diabetes mellitus, any inflammatory conditions, and cardiovascular disease. Articles were separated in two categories: genetic variants associated with either aerobic or resistance trainability ( $\triangleright$ Tables 1 and $\triangleright 2$ ). This review was conducted in accordance with the IJSM's ethical standards of the journal [28]

Finally, we selected SNPs that were classified as robust and separated them according to whether they were related to the aerobic trainability or resistance trainability. We chose this criteria as it reflects the reliability of the findings and increases the likelihood that there is true association of the SNP with trainability [27]. It also allows us to identify and summarise SNPs with biological relevance which is useful for researchers to 'select' candidate SNPs to identify causality and purpose of gene [29].

SNPs were considered robust if:

1) Consistent association with a given phenotype in at least two independent cohorts.
2) SNPs were shown to have functional relevance in an animal model or cell culture, with gene expression/DNA methylation Quantitative Trait Loci (QTLs) analysis or network, and enrichment analysis.

\section{Aerobic Trainability}

Twin and family studies indicate that $\sim 22-57 \%$ of aerobic fitness variability between individuals can be explained by genetics and therefore plays an important role in the range of aerobic phenotypes observed in a population [30]. Here, we briefly describe some of the robust SNPs that have been associated with aerobic trainability, which means they were replicated in at least 2 independent cohorts and were shown to have functional relevance.

A bioinformatic analysis study conducted by Ghosh et al. found that the greatest number of SNPs were annotated to the PPAR signalling pathway suggesting its importance in $\mathrm{VO}_{2 \max }$ trainability [31]. As such the most widely studied genes within this pathway are the peroxisome proliferator-activated receptors (PPARA, PPARG, and PPARD) and their transcriptional coactivators (PPARGC1A and PPARGC1B). These genes have been linked to multiple aerobic phenotypes, including muscle morphology, aerobic capacity and endurance performance [32,33]. PPARD is expressed predominantly in adipocytes and skeletal muscle where it promotes fatty acid oxidation [34]. In the HERITAGE family study, the rs2016520 SNP (C allele) located in PPARD was associated with reduced $\mathrm{VO}_{2 \max }$ and maximal power output after a 20 week endurance training intervention in African-Americans but not in Caucasians [35]. In vitro and animal studies show that the minor allele (C allele) in this SNP (rs2016520) results in higher PPARD transcriptional activity, which in turn promotes lipid accumulation and the alters normal regulation of lipid uptake and storage [34, 36, 37]. In a European cohort it was shown that the PPARD rs2267668 SNP was associated with $\mathrm{VO}_{\text {2peak }}$ and anaerobic threshold after a 9-month lifestyle intervention [38]. They then confirmed that in human primary cell lines that those carrying the minor allele at rs2267668 (G allele) were associated with lower mitochondrial activity, demonstrating a potential functional effect [38]. Taken together, PPARD locus may play a role in aerobic trainability, but larger cohorts of different ancestries and, more in depth functional studies to determine causal SNP are needed to confirm this.

The transcriptional co-activator PPARGC1A interacts with PPARD and regulates mitochondrial biogenesis, angiogenesis, lipolysis and adipogenesis [39]. Four candidate gene studies, predominantly in men, found consistent associations of rs 8192678 within PPARGC1A and aerobic capacity in Europeans [38, 40-42]. While in the Han Chinese cohort another nearby SNP (rs6821591) was associated with $\mathrm{VO}_{2 \max }$ specifically, the $\mathrm{G}$ allele was associated with increased $\mathrm{VO}_{2 \max }$ compared to those carrying the A allele [43]. Work conducted in a Han Chinese cohort found that the PPARGC1A rs6821591 SNP had functional significance as gene expression was altered and this was dependent on genotype ( $\mathrm{v} v \mathrm{G}$ allele) with the $\mathrm{G}$ allele displaying increased PGC-1 $\alpha$ gene expression [44]. Overexpression of PGC- $1 \alpha$ in an animal model showed increased Type 1 fibres in muscles that are normally Type II fibre type dense and this induced increases in resistance to fatigue, inferring increased aerobic capacity [45]. These population-specific results indicate that it is the 


\begin{tabular}{|c|c|c|c|c|c|}
\hline 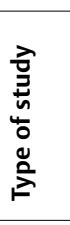 & 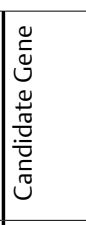 & 产 & 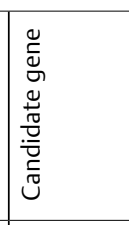 & 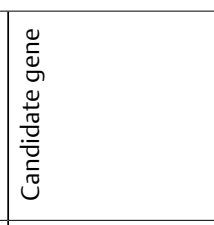 & 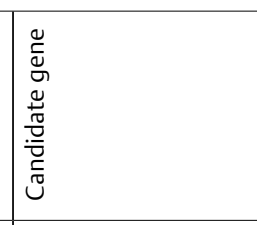 \\
\hline 总总 & 离产 & 崖 & 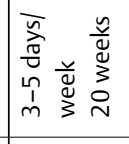 & 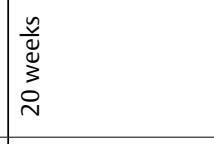 & 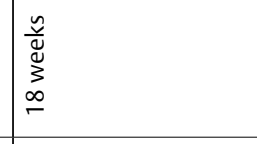 \\
\hline & 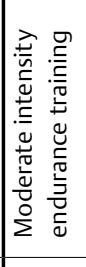 & 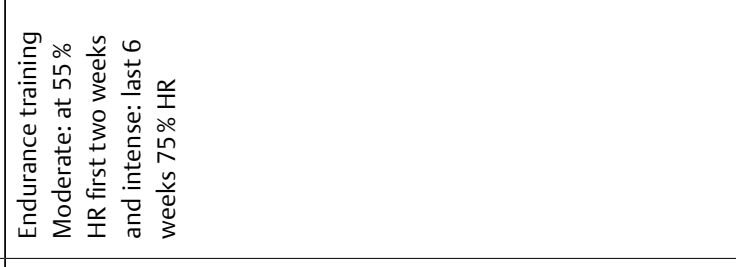 & 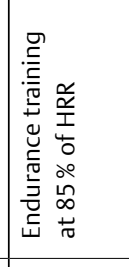 & 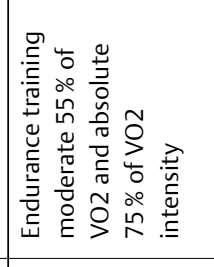 & 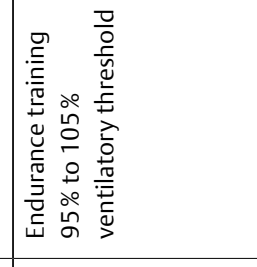 \\
\hline & 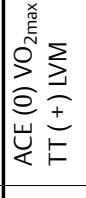 & 希 & 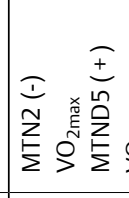 & 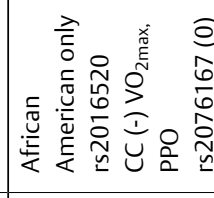 & 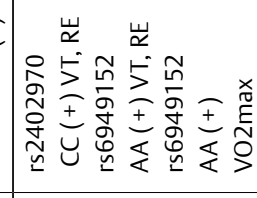 \\
\hline 㡍 & 瞏总 & 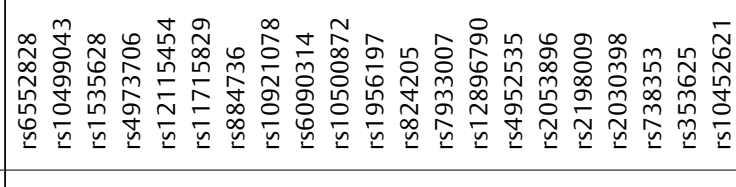 & & 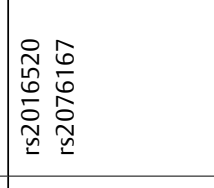 & 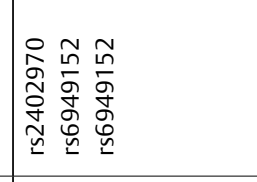 \\
\hline 总 & 蓆定 & 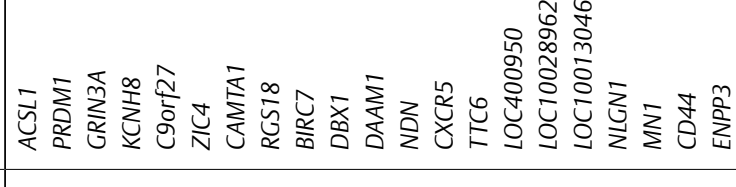 & 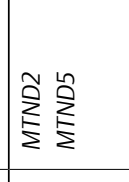 & 产 & 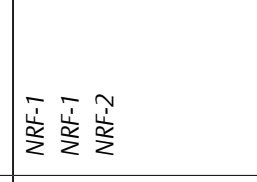 \\
\hline 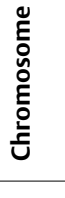 & $=-$ & 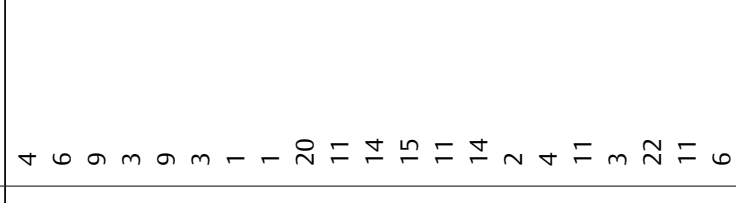 & 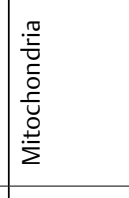 & $\approx$ & $\stackrel{2}{\sim}$ \\
\hline 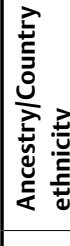 & 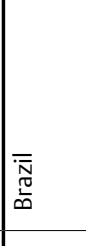 & 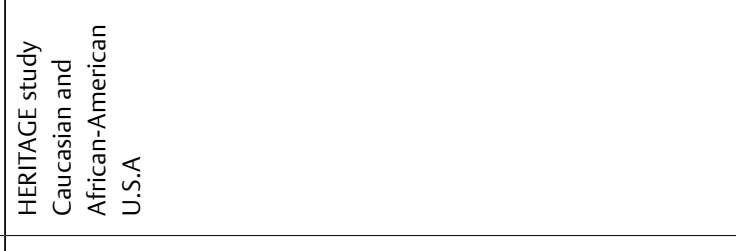 & 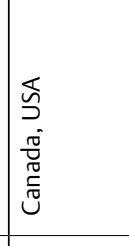 & 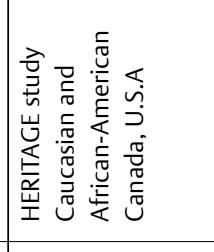 & 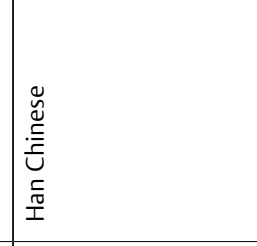 \\
\hline 娄 & 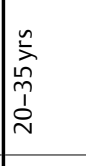 & 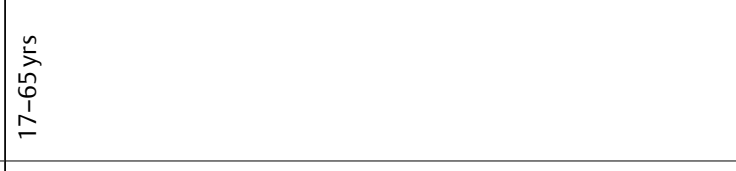 & 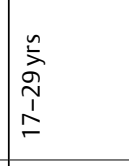 & 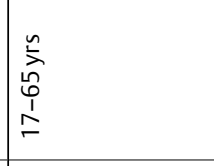 & 苛 \\
\hline 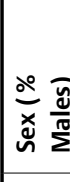 & 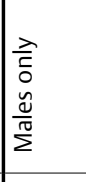 & 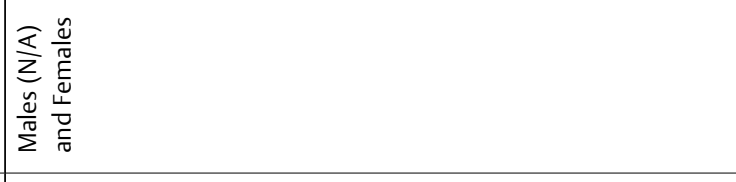 & 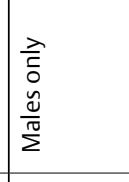 & 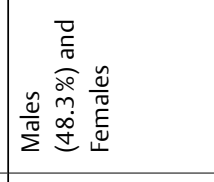 & 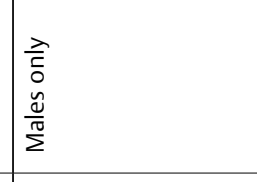 \\
\hline 焉总 & 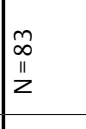 & $\begin{array}{l}\frac{7}{7} \\
\frac{\pi}{2}\end{array}$ & 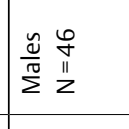 & $\mid \begin{array}{l}\infty \\
\substack{0 \\
z \\
z}\end{array}$ & $\mid \frac{\frac{\infty}{2}}{\frac{\pi}{2}}$ \\
\hline 崖 & $\mid$ & $\mid$ & 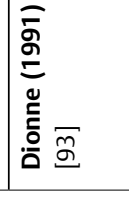 & 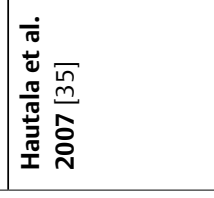 & 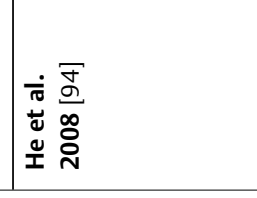 \\
\hline
\end{tabular}




\begin{tabular}{|c|c|c|c|c|c|c|c|c|c|}
\hline 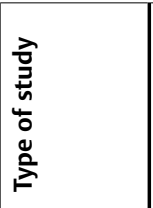 & 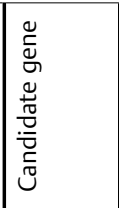 & 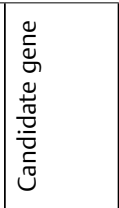 & 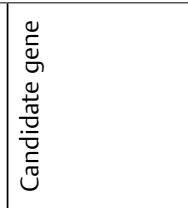 & 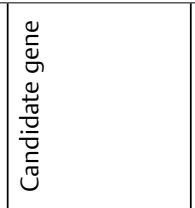 & 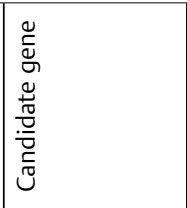 & 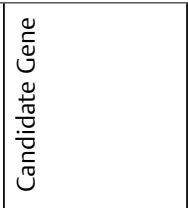 & 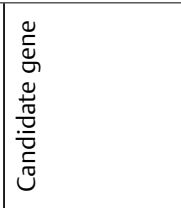 & 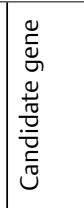 & 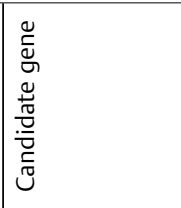 \\
\hline 它 & 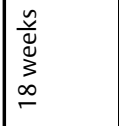 & 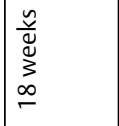 & 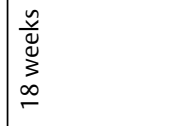 & 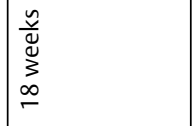 & 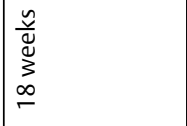 & 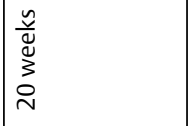 & 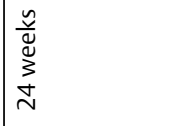 & 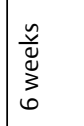 & 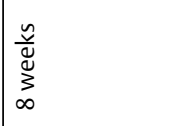 \\
\hline 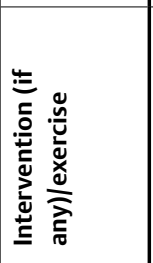 & 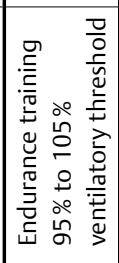 & 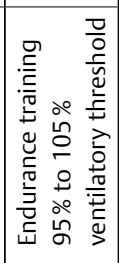 & 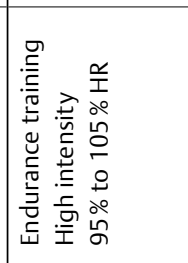 & 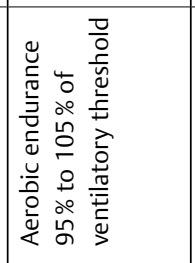 & 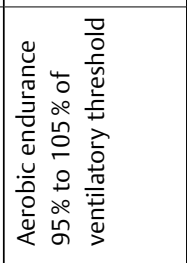 & 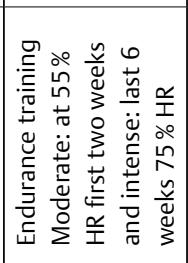 & 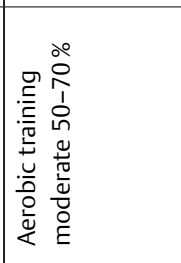 & 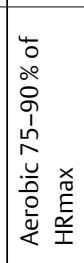 & 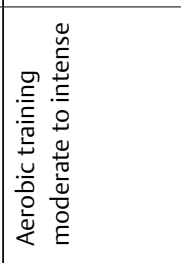 \\
\hline 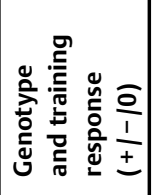 & 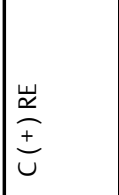 & 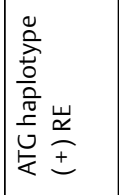 & 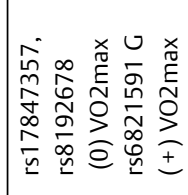 & 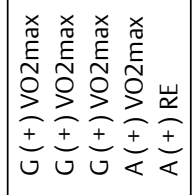 & 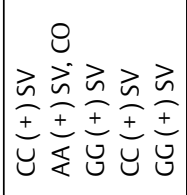 & 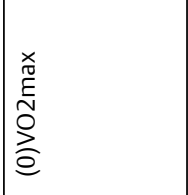 & 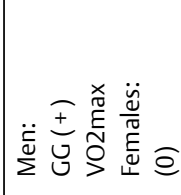 & 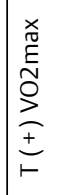 & 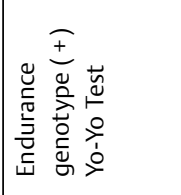 \\
\hline 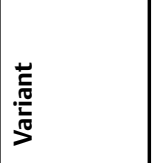 & 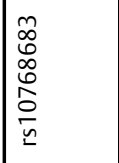 & 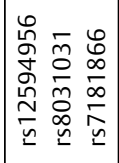 & 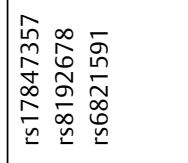 & 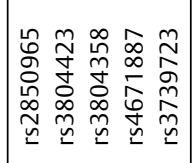 & 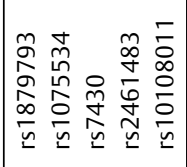 & $\begin{array}{l}\tilde{J} \\
\tilde{\omega} \\
\tilde{\omega} \\
\tilde{\omega}\end{array}$ & $\begin{array}{l}\frac{\sigma}{\tilde{N}} \\
\bar{m} \\
\frac{\tilde{n}}{\bar{n}}\end{array}$ & 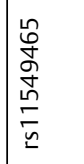 & 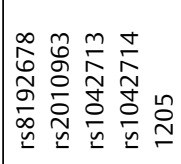 \\
\hline 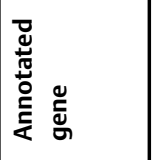 & 咅 & 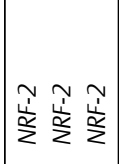 & 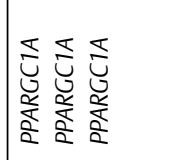 & 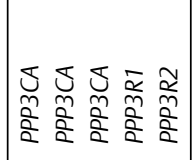 & 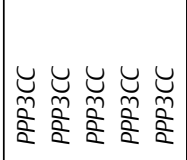 & \begin{tabular}{|l} 
岁 \\
$\grave{<}$
\end{tabular} & $\underset{⿱ 亠 䒑}{*}$ & 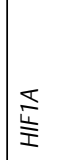 & 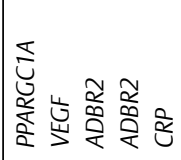 \\
\hline 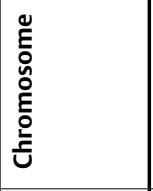 & $\mp$ & $\stackrel{\llcorner}{\llcorner}$ & $\gamma \nabla \gamma$ & $\nabla \nabla \nabla \sim a$ & $\infty \infty_{\infty} \quad \infty \quad \infty \quad \infty$ & 9 & I & \pm & $\nabla$ \\
\hline 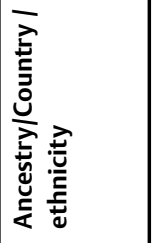 & 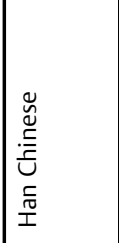 & 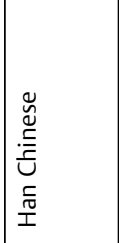 & 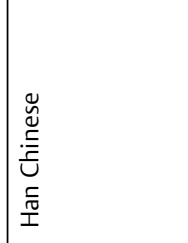 & 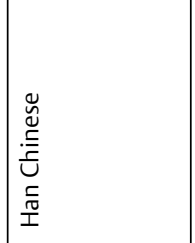 & 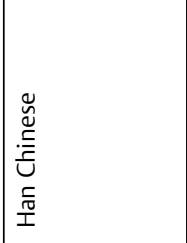 & 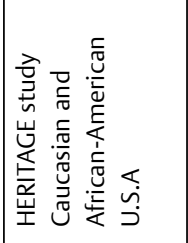 & 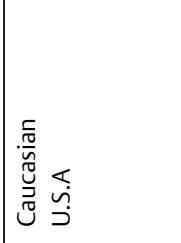 & 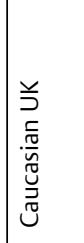 & 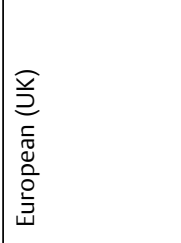 \\
\hline 芩 & $\begin{array}{l}+ \\
+ \\
\sigma \\
\sigma\end{array}$ & $\begin{array}{l}+ \\
\stackrel{+}{9} \\
\sigma\end{array}$ & $\begin{array}{l}+ \\
\stackrel{+1}{2} \\
\sigma\end{array}$ & $\begin{array}{l}+ \\
\stackrel{+}{g} \\
\sigma\end{array}$ & \begin{tabular}{l}
+ \\
$\stackrel{1}{2}$ \\
\hdashline
\end{tabular} & 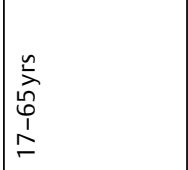 & 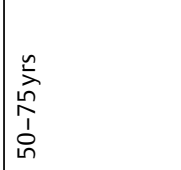 & 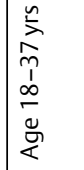 & 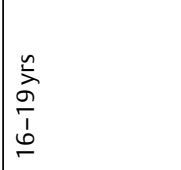 \\
\hline 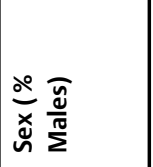 & 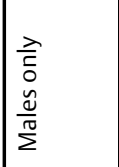 & 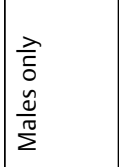 & 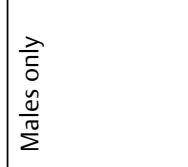 & 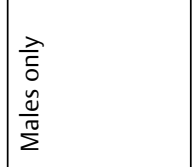 & 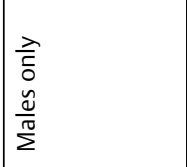 & 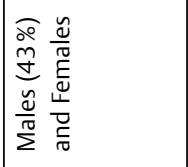 & 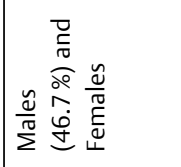 & 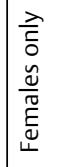 & 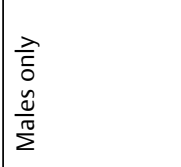 \\
\hline 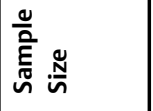 & $\begin{array}{l}\bar{\infty} \\
\overline{11} \\
z\end{array}$ & $\begin{array}{l}\bar{\infty} \\
\overline{11} \\
z\end{array}$ & $\begin{array}{l}\bar{\infty} \\
\overline{11} \\
z\end{array}$ & $\begin{array}{l}\infty \\
\infty \\
\bar{z} \\
z\end{array}$ & $\begin{array}{l}\bar{\infty} \\
\frac{1}{21} \\
z\end{array}$ & $\begin{array}{l}\ell \\
\stackrel{2}{2} \\
11 \\
z\end{array}$ & $\begin{array}{l}8 \\
\frac{9}{11} \\
2\end{array}$ & $\begin{array}{l}\infty \\
\omega \\
\text { II } \\
z\end{array}$ & $\begin{array}{l}\tilde{y} \\
\text { II }\end{array}$ \\
\hline 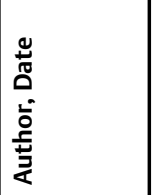 & 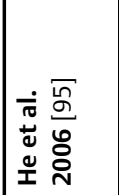 & 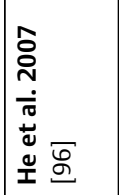 & 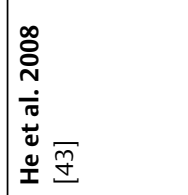 & 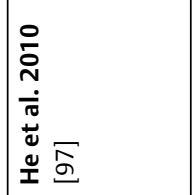 & 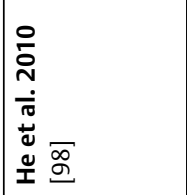 & 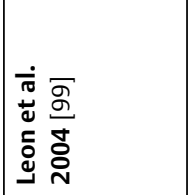 & 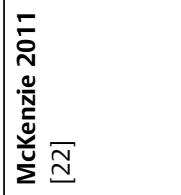 & 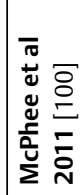 & 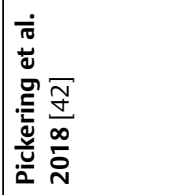 \\
\hline
\end{tabular}




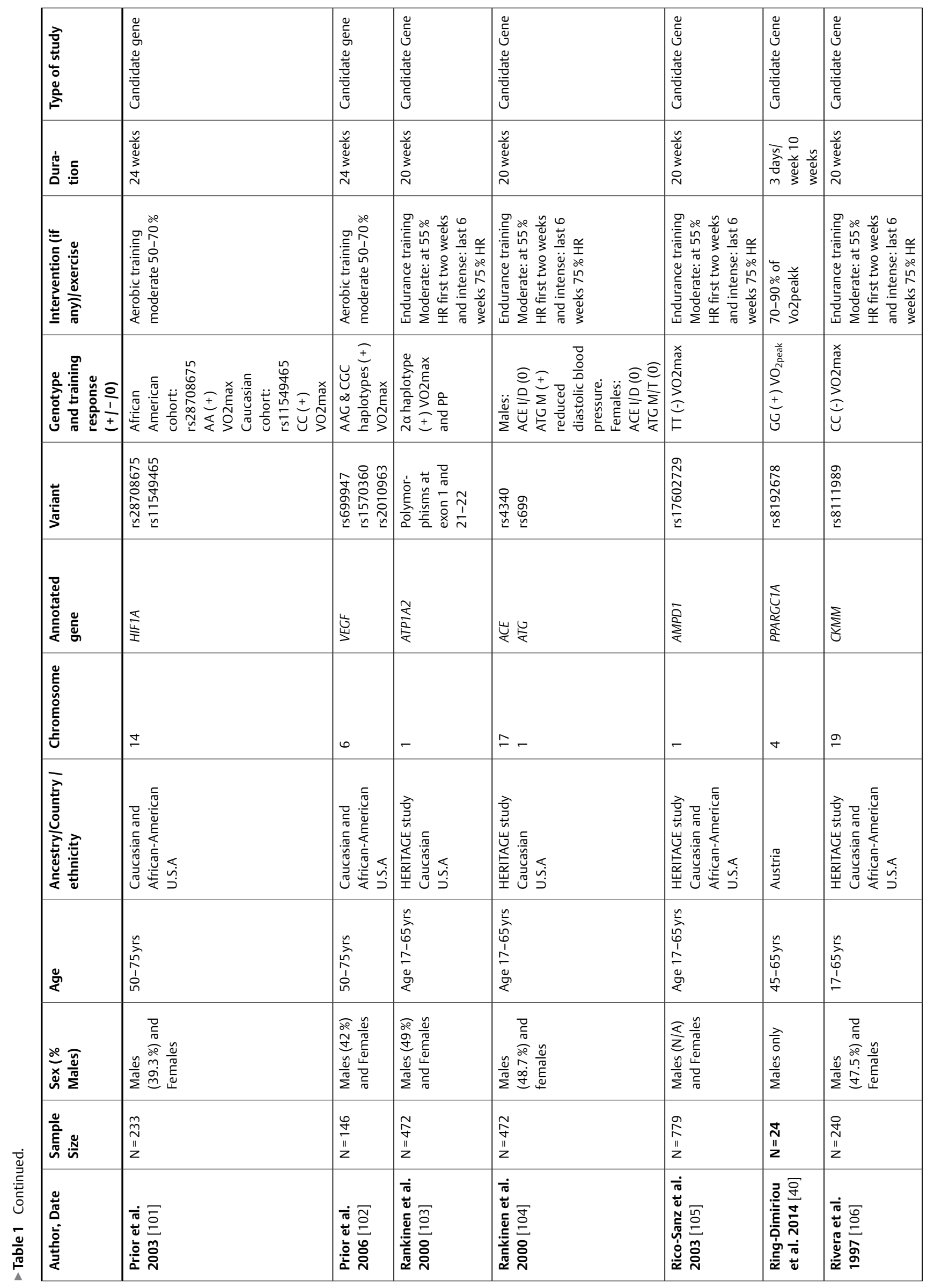




\begin{tabular}{|c|c|c|c|c|c|c|c|c|}
\hline 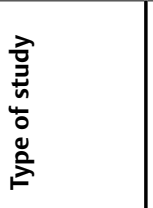 & 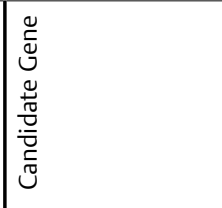 & 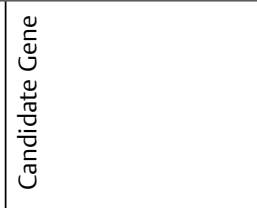 & 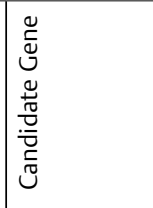 & 柋 & 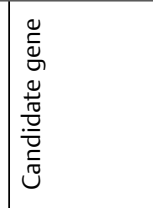 & 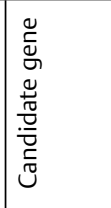 & 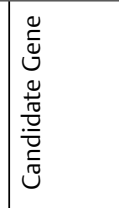 & ह் \\
\hline 窝 & 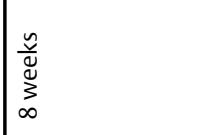 & 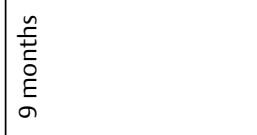 & 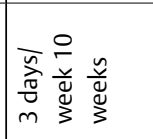 & $\begin{array}{l}\frac{\tilde{v}}{\tilde{\Xi}} \\
\sum_{a} \\
a\end{array}$ & 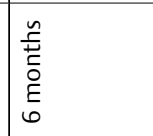 & 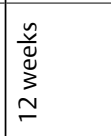 & 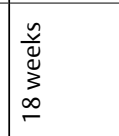 & 总 \\
\hline 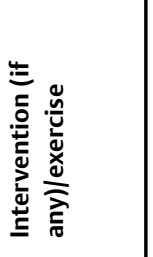 & 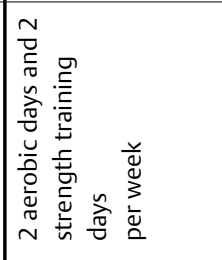 & 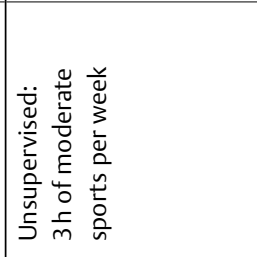 & 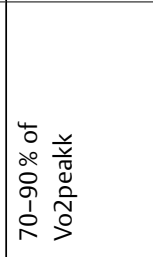 & 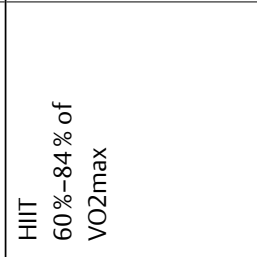 & 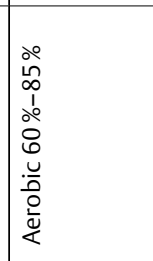 & 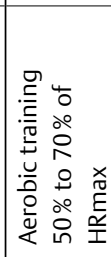 & 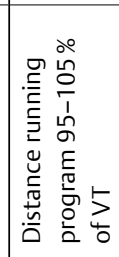 & \\
\hline 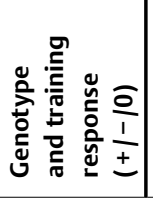 & 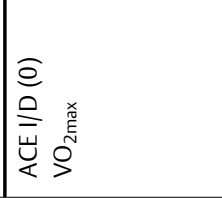 & 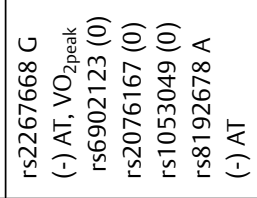 & 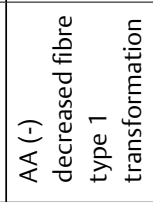 & 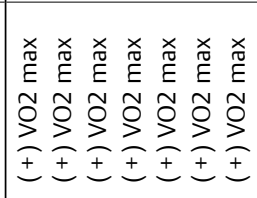 & 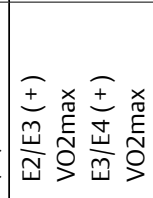 & 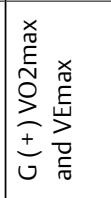 & 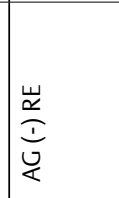 & 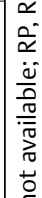 \\
\hline 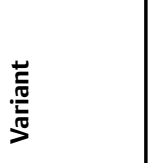 & 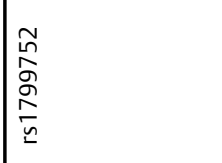 & 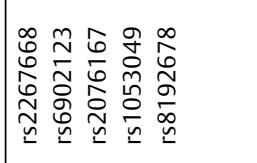 & $\begin{array}{l}\infty \\
0 \\
\omega \\
o \\
\infty \\
\infty \\
0 \\
\end{array}$ & 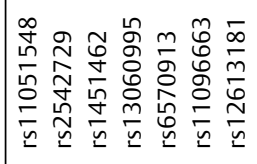 & 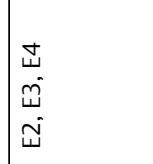 & 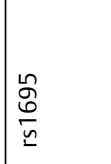 & $\begin{array}{l}n \\
\infty \\
\widetilde{N} \\
\infty \\
\infty \\
\tilde{n}\end{array}$ & . \\
\hline 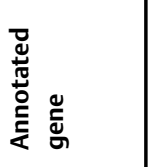 & 亗 & 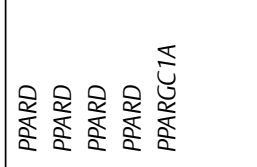 & 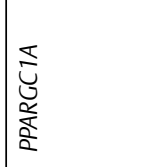 & 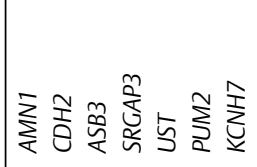 & 岁 & $\overline{\frac{2}{5}}$ & $\sum_{\bar{\Sigma}}^{\sum}$ & \\
\hline 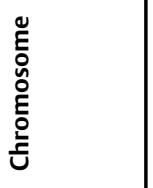 & $\approx$ & $\approx N \approx \approx+$ & $\theta$ & $\simeq \stackrel{\infty}{\sim} \sim m \cup \sim \sim$ & 9 & $=$ & 9 & 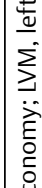 \\
\hline 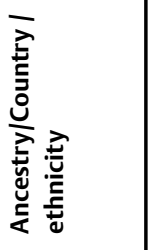 & 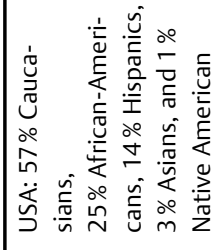 & 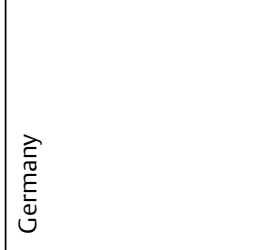 & 总 & 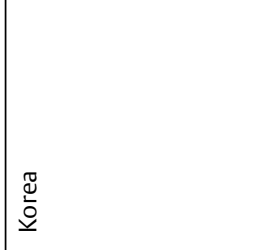 & 胥 & 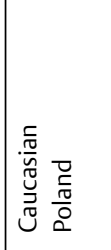 & 胥 & 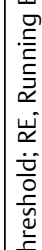 \\
\hline 荧 & 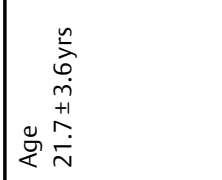 & 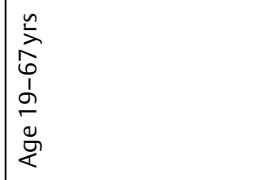 & \begin{tabular}{|l}
$n$ \\
\\
0 \\
0 \\
0 \\
0 \\
nn
\end{tabular} & 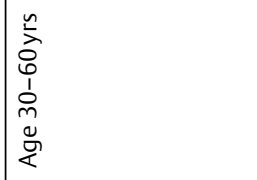 & 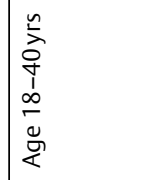 & 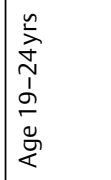 & $\begin{array}{l}n \\
\\
o \\
0 \\
+1 \\
\infty+1 \\
\infty \\
\infty \\
\infty\end{array}$ & 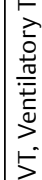 \\
\hline 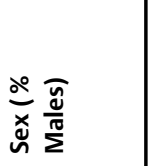 & 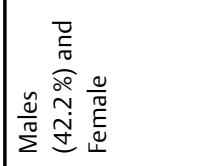 & 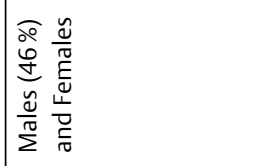 & 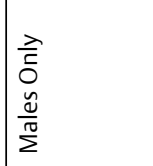 & 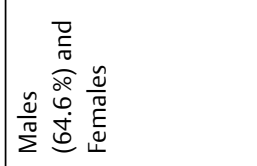 & 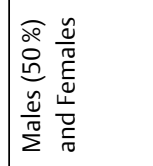 & 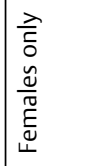 & 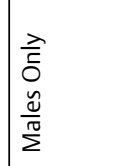 & 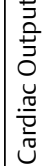 \\
\hline 号 & $\begin{array}{l}\text { f } \\
\bar{z} \\
z\end{array}$ & $\begin{array}{l}0 \\
m \\
11 \\
z\end{array}$ & $\begin{array}{l}\infty \\
\mathbb{N} \\
2\end{array}$ & $\begin{array}{l}9 \\
11 \\
z\end{array}$ & $\begin{array}{l}8 \\
0 \\
11 \\
z\end{array}$ & $\begin{array}{l}0 \\
l 1 \\
z\end{array}$ & $\begin{array}{l}0 \\
0 \\
\pi \\
z\end{array}$ & $\frac{\ddot{\theta}}{0}$ \\
\hline 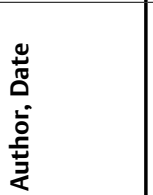 & 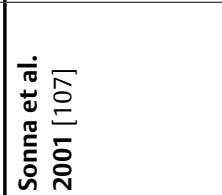 & 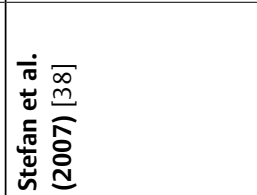 & 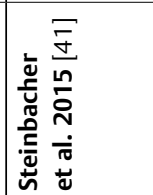 & 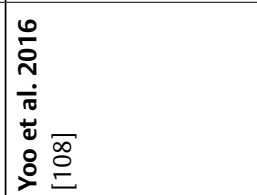 & 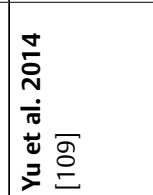 & 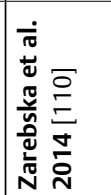 & 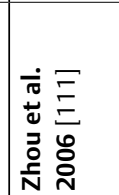 & 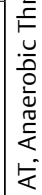 \\
\hline
\end{tabular}




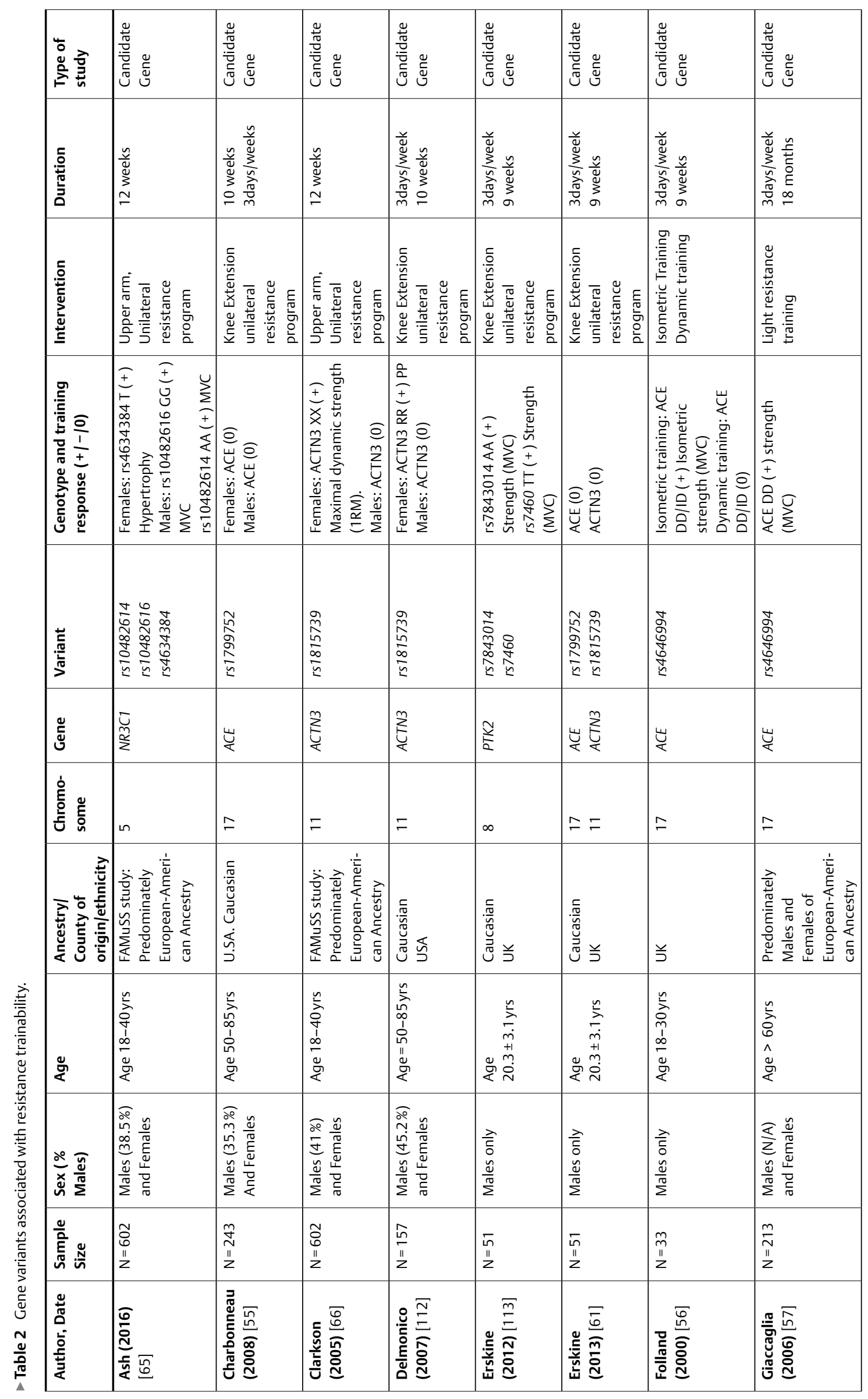




\begin{tabular}{|c|c|c|c|c|c|}
\hline 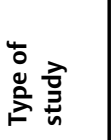 & 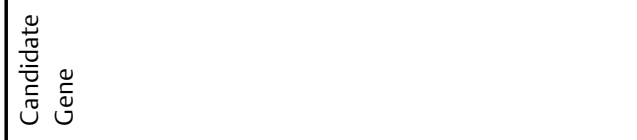 & 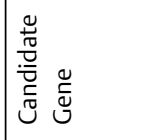 & 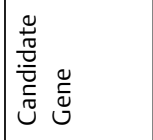 & 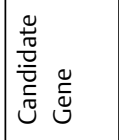 & 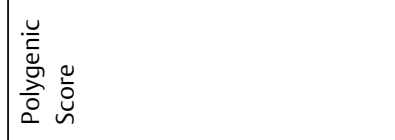 \\
\hline & 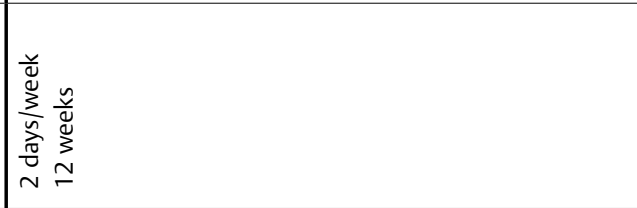 & 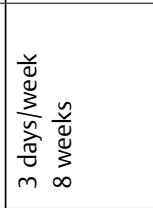 & 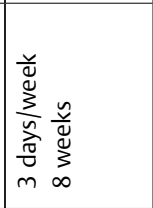 & 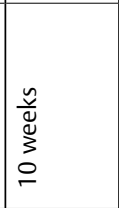 & 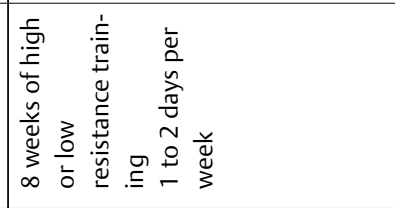 \\
\hline & 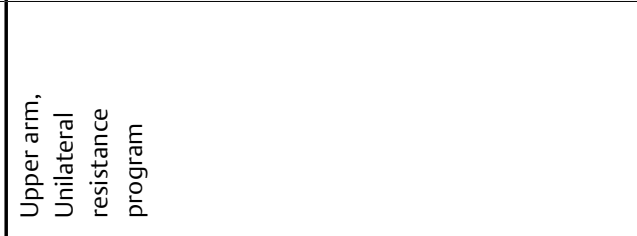 & 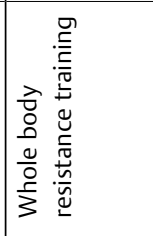 & 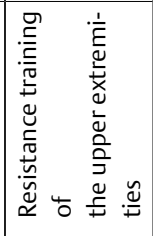 & 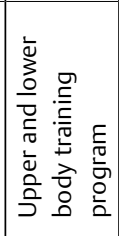 & 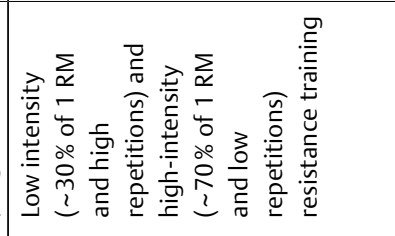 \\
\hline 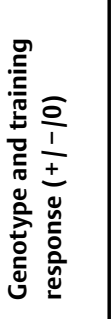 & 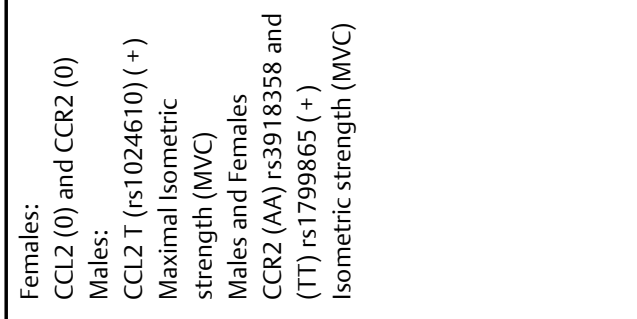 & 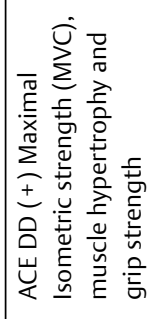 & 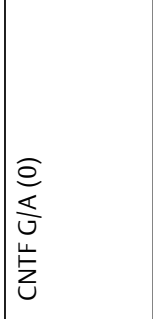 & 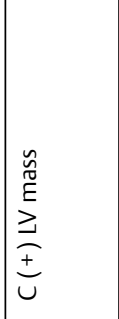 & 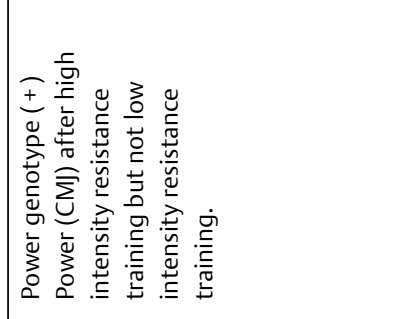 \\
\hline 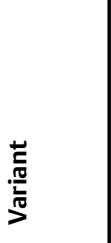 & 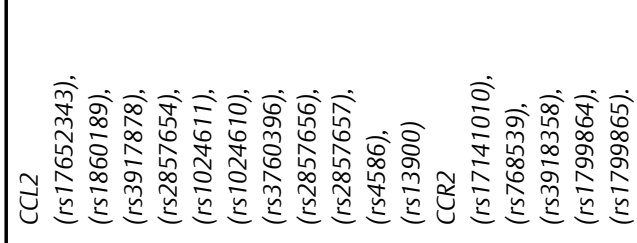 & 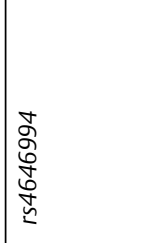 & $\begin{array}{l}0 \\
0 \\
0 \\
\infty \\
\frac{0}{2}\end{array}$ & 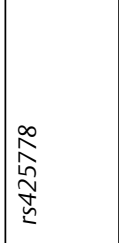 & 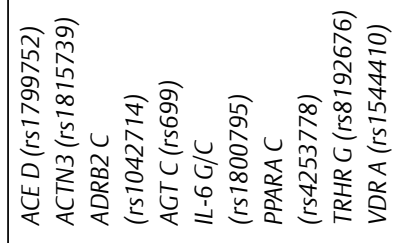 \\
\hline Ũّ & ปี & 岁 & 岁 & \begin{tabular}{|l}
$\frac{a}{2}$ \\
$\frac{2}{2}$ \\
$\frac{a}{2}$
\end{tabular} & 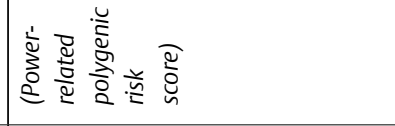 \\
\hline 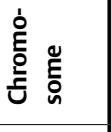 & $\neq m$ & $\stackrel{F}{ }$ & $=$ & 0 & $\neq=$ \\
\hline 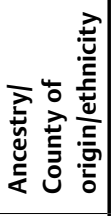 & 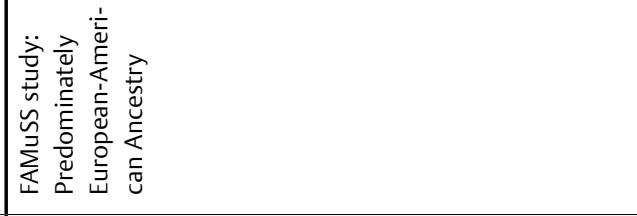 & 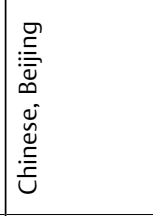 & 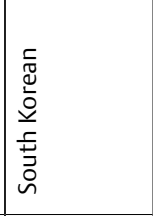 & כ & 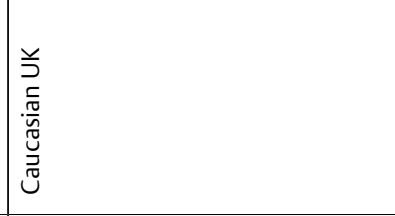 \\
\hline 苂 & 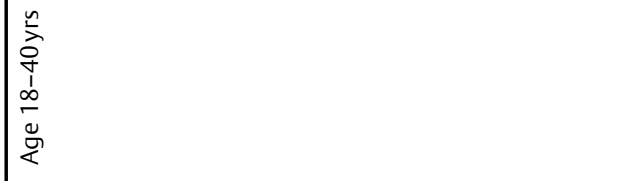 & 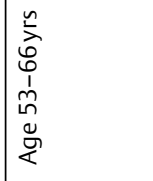 & 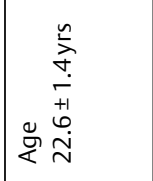 & 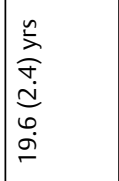 & 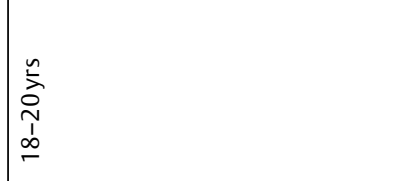 \\
\hline 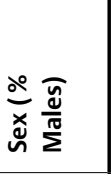 & 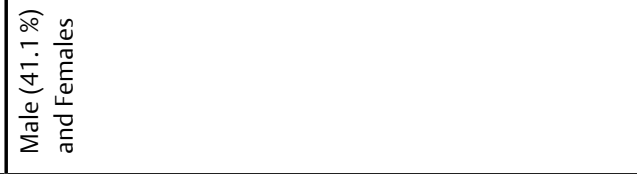 & 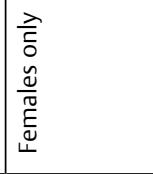 & 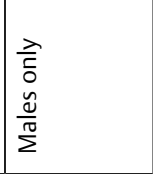 & 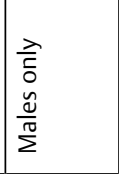 & 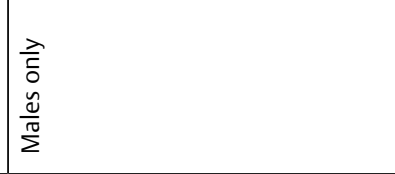 \\
\hline 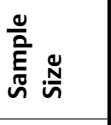 & 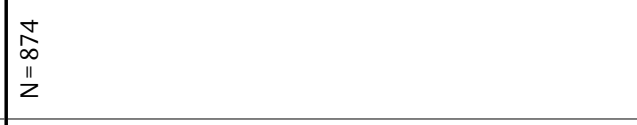 & $\begin{array}{l}\text { P } \\
11 \\
z\end{array}$ & $\begin{array}{l}m \\
\infty \\
\| 1 \\
z\end{array}$ & $\begin{array}{l}\frac{J}{J} \\
\frac{\pi}{2}\end{array}$ & 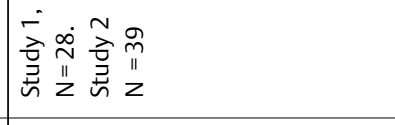 \\
\hline 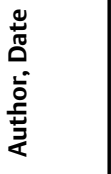 & 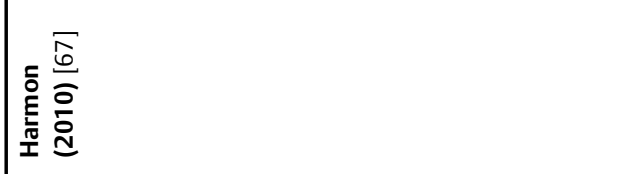 & 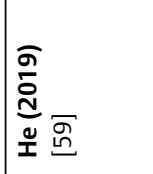 & 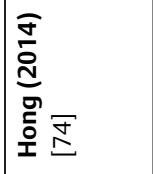 & 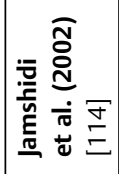 & 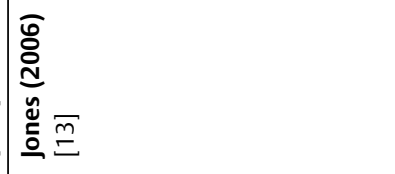 \\
\hline
\end{tabular}




\begin{tabular}{|c|c|c|c|c|c|c|c|c|}
\hline | & 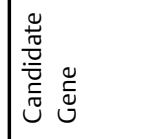 & 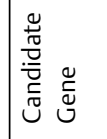 & 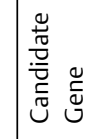 & 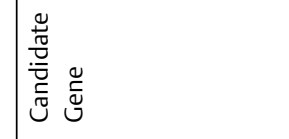 & 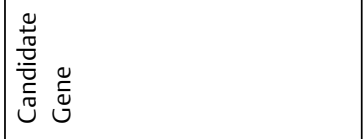 & 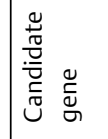 & 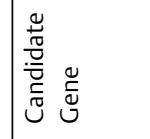 & 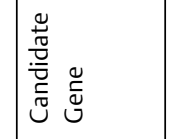 \\
\hline 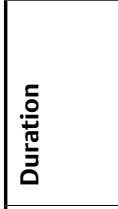 & 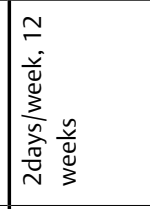 & 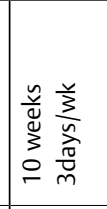 & 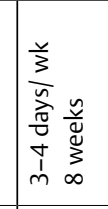 & 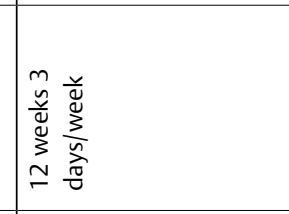 & 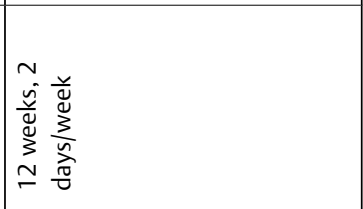 & 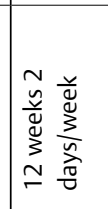 & 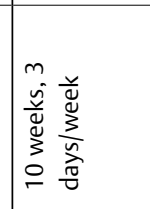 & 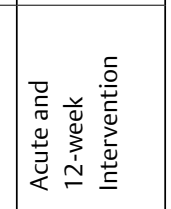 \\
\hline 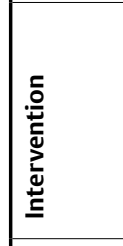 & 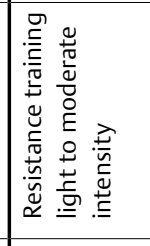 & 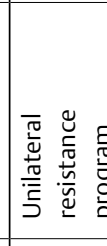 & 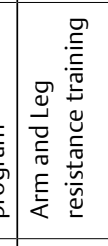 & 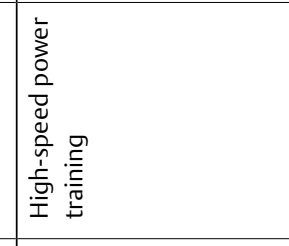 & 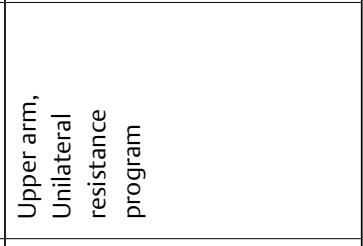 & 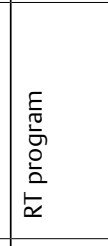 & 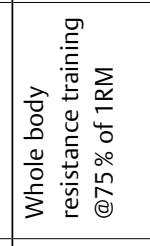 & 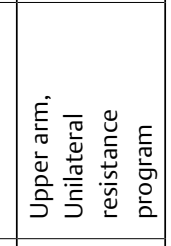 \\
\hline 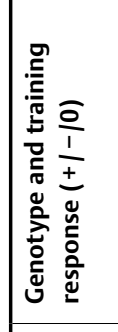 & 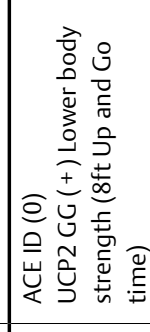 & 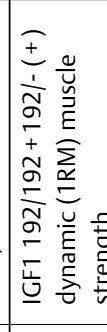 & 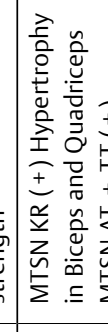 & 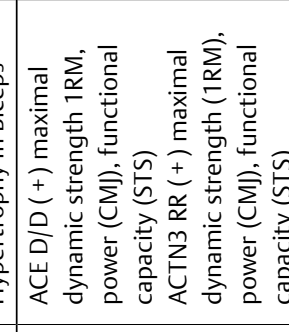 & 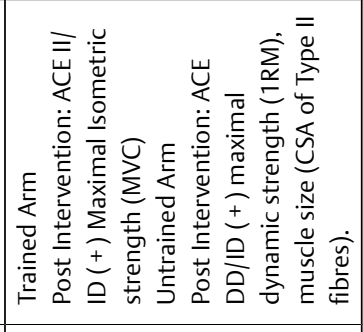 & 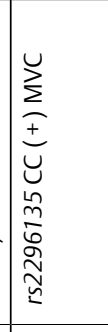 & 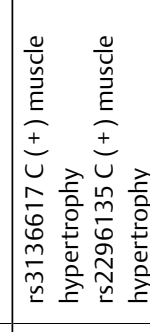 & 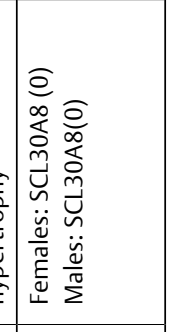 \\
\hline 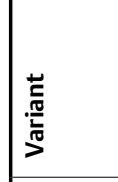 & 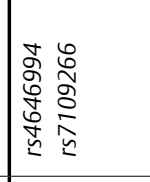 & 总 & 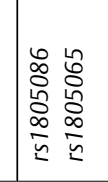 & 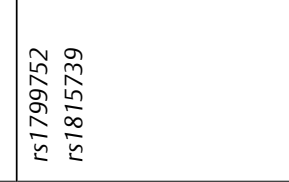 & 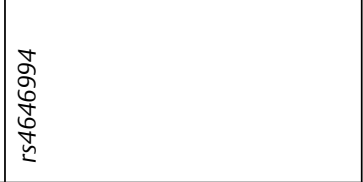 & $\mid$ & 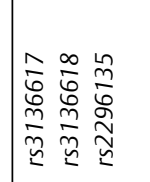 & 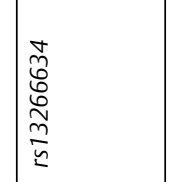 \\
\hline 遌 & चू ड̃ & E & 产 & 岁衡 & 岁 & 䍃 & 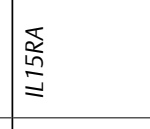 & 然 \\
\hline 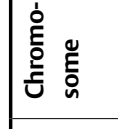 & $=$ & $\simeq$ & N & $\neq=$ & $=$ & $\cong$ & $\because$ & $\infty$ \\
\hline 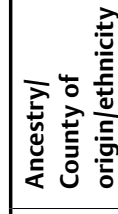 & 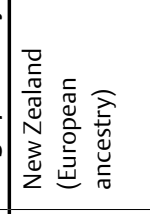 & 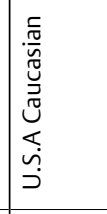 & 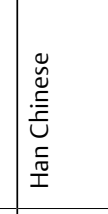 & 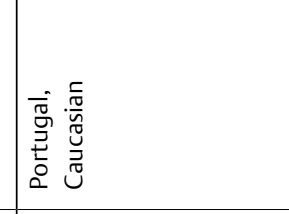 & 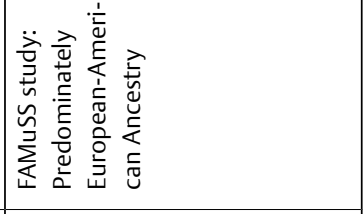 & 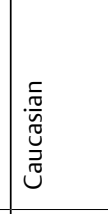 & 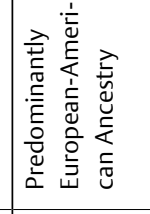 & 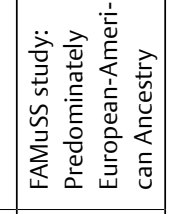 \\
\hline 冚 & 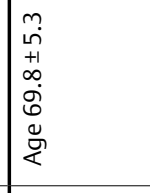 & 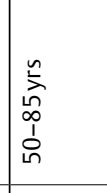 & 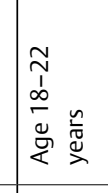 & 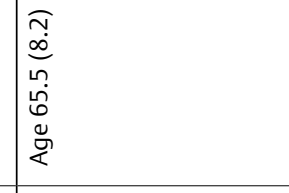 & 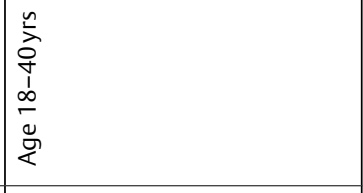 & 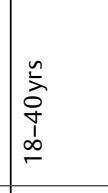 & 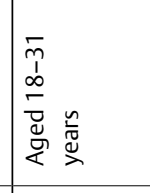 & 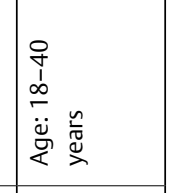 \\
\hline 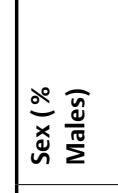 & 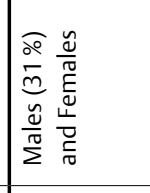 & 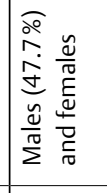 & 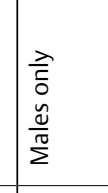 & 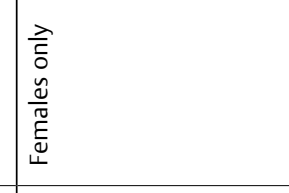 & 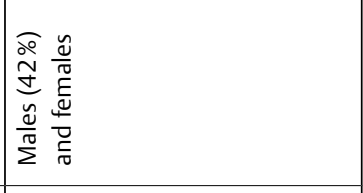 & 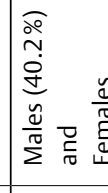 & 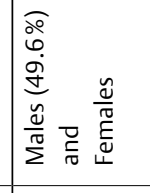 & 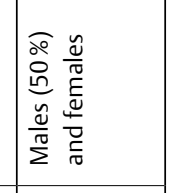 \\
\hline 总密 & 变 & i & 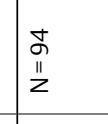 & $\frac{\frac{\rho}{2}}{\frac{m}{2}}$ & \begin{tabular}{|l}
$\overline{\tilde{O}}$ \\
$\bar{z}$ \\
$z$
\end{tabular} & \begin{tabular}{|l}
$\infty$ \\
0 \\
$\vdots$ \\
$z$ \\
$z$
\end{tabular} & $\frac{\frac{m}{2}}{\frac{\pi}{2}}$ & 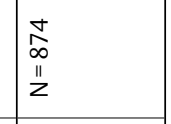 \\
\hline 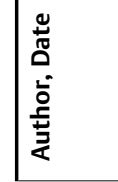 & 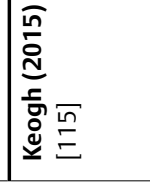 & 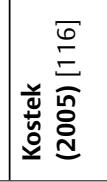 & 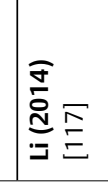 & 离 & 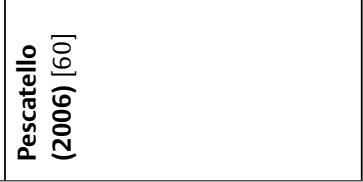 & 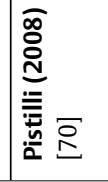 & 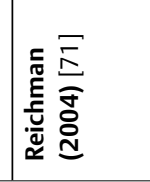 & 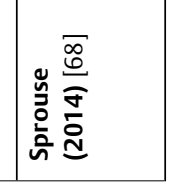 \\
\hline
\end{tabular}




\begin{tabular}{|c|c|c|c|c|}
\hline $\begin{array}{l}\text { 흐 } \\
\vdots \\
\stackrel{0}{2}\end{array}$ & 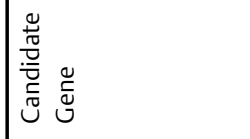 & 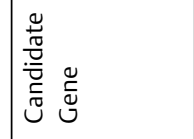 & 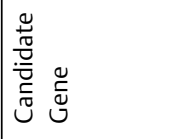 & 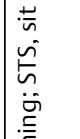 \\
\hline 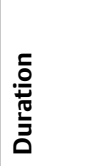 & 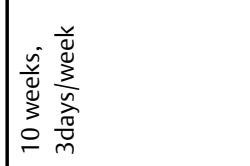 & 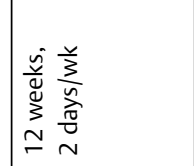 & 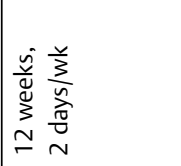 & 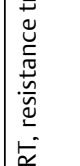 \\
\hline 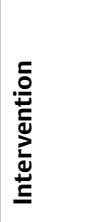 & 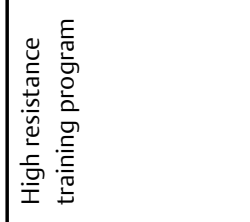 & 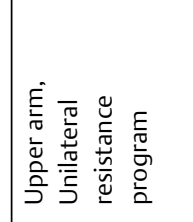 & 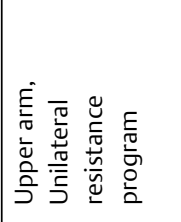 & 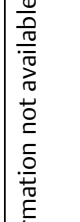 \\
\hline 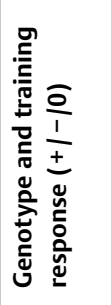 & 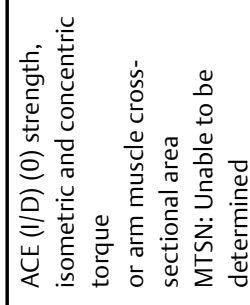 & 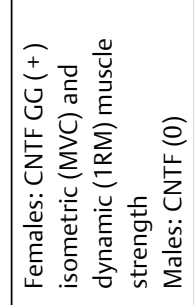 & 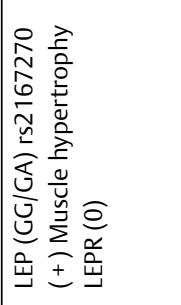 & 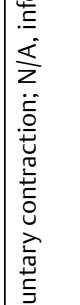 \\
\hline 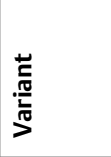 & 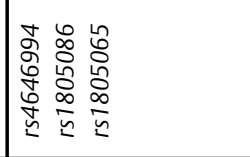 & $\begin{array}{l}0 \\
0 \\
0 \\
0 \\
\vdots \\
2\end{array}$ & 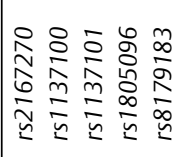 & 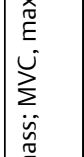 \\
\hline 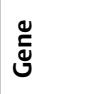 & 崖 & 岕 & 总总 & 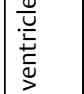 \\
\hline 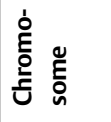 & $\approx N$ & $=$ & - & 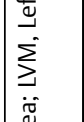 \\
\hline 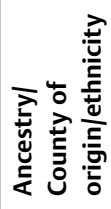 & 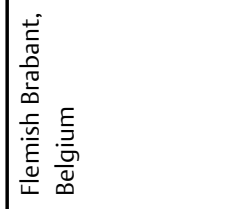 & 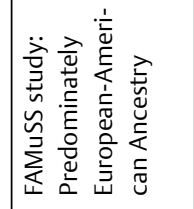 & 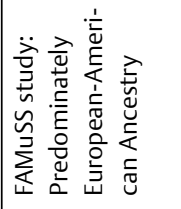 & 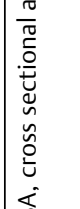 \\
\hline 娄 & 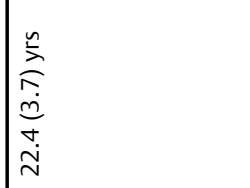 & 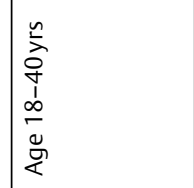 & 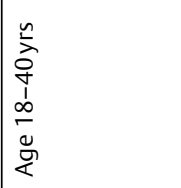 & 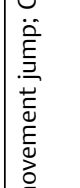 \\
\hline 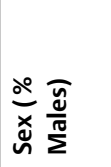 & 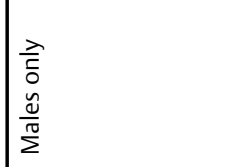 & 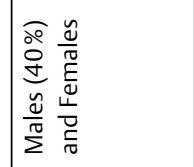 & 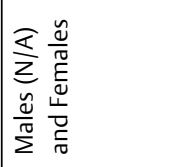 & 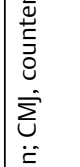 \\
\hline 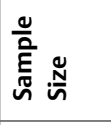 & $\begin{array}{l}n \\
n \\
\text { z }\end{array}$ & $\begin{array}{l}\frac{1}{f} \\
\text { II } \\
z\end{array}$ & $\begin{array}{l}8 \\
11 \\
11 \\
z\end{array}$ & 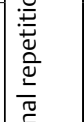 \\
\hline 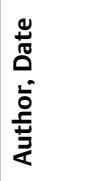 & 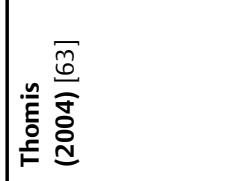 & 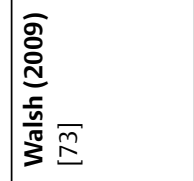 & 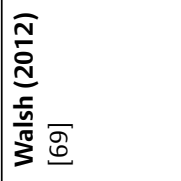 & 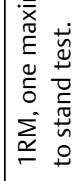 \\
\hline
\end{tabular}


PPARGC1A locus itself, rather than individual SNPs located within that locus, may be important for trainability $[43,46]$.

Currently 26 SNPs associated with $\mathrm{VO}_{2 \max }$ trainability were identified in a GWAS and were validated in 2 separate cohorts (detailed in $>$ Table 2) [23]. They accounted for $49 \%$ of $\mathrm{VO}_{2 \max }$ trainability and were able to classify responders and non-responders $[23,47]$. Whether these SNPs are directly involved in gene function or regulation of genes is the next step to validate these findings. The most robust is the SNP rs6552828 located near the ACSL1 gene which was the strongest predictor ( $~ 6 \%$ ) of aerobic trainability $\left(\mathrm{VO}_{2 \max }\right)$ [23]. It has subsequently been validated in a bioinformatics pathway analysis and found to be strongly correlated to the aerobic electron transport chain phenotype and the PPAR signalling pathway providing a robust candidate gene in $\mathrm{VO}_{2 \max }$ trainability [31]. ACSL1 regulates lipid metabolism by facilitating the transport of long chain fatty acids into the mitochondria and is an essential step in fatty acid oxidation [48]. Timmons et al. integrated RNA profiles with genetic variants and found the following genes $C D 44$, and $D A A M 1$, also discovered in the Bouchard et al. GWAS, were associated with gene expression changes [49]. Gene expression of CD44 was up-regulated in response to endurance training [49] and was strongly associated with phenotypic terms associated with aerobic exercise such as: cardiovascular physiological processes, muscle contraction, physical fitness and aerobic electron transport chain [31] indicating that this gene and any alterations to its function (i. e. via SNPs) may play in important role in aerobic trainability. While these genes certainly provide robust genes, there are still limitations in determining the causality of these particular SNPs in the molecular mechanisms affecting aerobic trainability.

Many candidate gene and GWAS studies have been conducted and this review highlights the large collection of candidate genes that have been associated with aerobic trainability. Only 12 SNPs have been robustly associated with aerobic trainability ( $>$ Table 3 ) meaning that have been validated in at least 2 independent cohorts and were shown to have some functional relevance. Subsequent studies should focus on understanding the functional role of the SNPs that have been replicated as this review highlights the lack of understanding of the molecular mechanism and limits our understanding of aerobic trainability.

\section{Resistance Trainability}

Muscular strength and power show a heritability estimated around $52 \%$ [14]. Skeletal muscle strength is defined as the force produced by muscle contraction. A variety of measures have been investigated, including muscle strength, maximal voluntary contraction (MVC), 1 repetition maximum (1RM) and handgrip strength. While the production of skeletal muscle power is defined as how much force can be produced and the velocity at which it is produced. The production of power can be measured at the by undertaking tests such as Wingate's, counter movement jumps (CMJ) and vertical jumps (VJ).

The ACE I/D and ACTN3 R/X SNPs are two of the most extensively studied gene loci. We have chosen not to discuss ACTN3 here as it has recently been reviewed in detail by Del Coso et al. [50] and instead focus on the ACE I/D SNP. The ACE gene encodes the angiotensin-converting enzyme that is a central component of the renin-angiotensin-system [51]. The $A C E$ I/D results in either an insertion (I) or deletion (D) of a 287-basepair region in intron 16 of the gene [52] and can alter the levels of ACE in the blood [52]. It has recently been shown that the polymorphism can manipulate the activity of the $\mathrm{C}$ - and $\mathrm{N}$-terminal domain in the enzyme [53]. Further, exercise can decrease the enzyme activity in the C-terminal domain and increase the activity in the $\mathrm{N}$ - terminal domain which results in improved blood flow and proliferation of red blood cells [53]. It is thought that the I allele confers enhanced endurance performance while the $D$ allele is thought to confer increased muscle power and strength [54]. The D allele was consistently shown across 6 separate candidate gene studies to be associated with greater gains in strength after resistance training and this was consistent across sex and age [55-60]. While the literature is consistent regarding muscular strength, the association with muscular power is less convincing [55, 61-63]. The D allele in ACE was associated with CM] in older females after a 12-week power training program [58] and in young males after a high intensity training program [13]. However, it was the I allele in ACE that was associated with a higher

> Table 3 Robust SNPs associated with aerobic or resistance trainability.

\begin{tabular}{|c|c|c|c|c|c|}
\hline \multicolumn{3}{|c|}{ Aerobic trainability } & \multicolumn{3}{|c|}{ Resistance trainability } \\
\hline SNP & Nearest gene & Beneficial allele & SNP & Nearest gene & Beneficial allele \\
\hline rs6552828 & ACSL1 & $\mathrm{G}$ & rs4646994* & $A C E$ & $\mathrm{D}$ \\
\hline rs699 & AGT & $\mathrm{T}$ & rs1799752* & ACE & $\mathrm{D}$ \\
\hline rs6090314 & $B I R C$ & A & rs4340* & ACE & $\mathrm{D}$ \\
\hline rs12580476 & C12orf36 & TBC & rs $13447447^{*}$ & ACE & $\mathrm{D}$ \\
\hline rs884736 & CAMTA1 & $\mathrm{G}$ & rs1815739 & ACTN3 & $R$ \\
\hline rs353625 & CD44 & TBC & rs2296135 & IL15RA & $\mathrm{C}$ \\
\hline rs1956197 & DAAM1 & $\mathrm{G}$ & rs4253778 & PPARA & C \\
\hline rs17117533 & $N D N$ & $\mathrm{~A}$ & & & \\
\hline rs8192678 & PPARGC1A & G & & & \\
\hline rs10921078 & RGS18 & $A$ & & & \\
\hline rs7531957 & RYR2 & TBC & & & \\
\hline rs11715829 & ZIC4 & G & & & \\
\hline
\end{tabular}


baseline V] at baseline in males and females [62]. Another two studies did not find any association between the ACE I/D and skeletal muscle power at baseline or in response to resistance training $[61,63]$. ACE provides a robust candidate gene for explaining variation in muscular strength but not muscular power suggesting that this gene loci may only explain some of the inter-individual resistance variability dependent on type of resistance exercise.

Many of the candidate genes in resistance trainability came from a large multi-centre trial (FAMuSS) which aimed to identify nonsynonymous SNPs with functional effects on muscle power and strength [64]. These include: Glucocorticoid receptor (NR3C1)[65], alpha-actinin 3 (ACTN3)[66], Chemokine (C-C motif) ligand 2 (CCL2)[67], Chemokine (C-C motif) ligand 2 Receptor (CCR2)[67], ACE[60], Solute carrier family 30 (zinc transporter), member eight gene (SLC30A8)[68], Leptin (LEP) and Leptin receptor (LEPR)[69]. The FAMuSS study was conducted in young (18-40 years old) males $(\mathrm{N}=247)$ and females $(\mathrm{N}=355)$ of predominantly European-American ancestry. Participants underwent a 12-week unilateral resistance program consisting of upper arm exercises in the non-dominant arm [60]. Only IL-15RA, ACTN3 and ACE from this series of studies were replicated in separate cohorts and have functional relevance. In the IL-15RA locus the rs2296135 SNP was associated gains in muscular strength and replicated in two different studies in cohort of European ancestry [70,71]. When the gene IL-15RA is knocked down in an animal model it altered the contractile properties and fatigability in skeletal muscle fibres [72]. While the locus is important it not yet clear which SNPs is responsible for altering the function of IL-15RA protein. Although SNPs within CCL2, CCR2 and CNTF have not been replicated they interestingly showed sexspecific associations with muscle strength. CTNF polymorphisms were associated with strength gains only in females [73], which was subsequently confirmed in a South Korean cohort [74]. SNPs in CCL2 and CCR2 were associated strength gains in males only [67]. This indicates potential sex-specific differences in the genetic architecture of complex traits and should be incorporated into study design $[75,76]$. In addition PTK2, CNTF, IL-6, PPARA and VDR candidate genes have been replicated with functional relevance $[13,73]$.

In total 7 SNPs ( $\vee$ Table 3 ) were robustly associated with resistance variability. While there are plethora of candidate gene studies no GWAS have been conducted that specifically focuses on resistance trainability.

\section{Functional Validation}

We have identified 12 SNPs and 7 SNPs that are robustly associated with variance in aerobic and resistance trainability respectively. The next steps are to a) identify the causal SNP, b) annotate the casual SNP to the correct gene and then c) to establish the functional relevance of the gene [47]. The overall evidence from literature connecting causal genes to trainability is relatively low [31]. If we hope to identify the casual variants or genes it is vital that we begin to integrate "omic" technologies from the genome and epigenome to transcriptome to proteome and metabolome which can capture a complete picture of complex human traits such as aerobic and resistance trainability $[77,78]$.

There have been attempts to associate molecular pathways or “molecular phenotypes" with physiological phenotypes of aerobic and resistance trainability [79-81]. Sarzynski et al. applied this systems biology approach by combining the 21 SNP identified in a GWAS from the HERITAGE study cohort ( amined the joint contributions of these SNPs to exercise response [47]. This approach identified potential pathways in calcium signalling, energy sensing and partitioning, mitochondrial biogenesis, angiogenesis, immune functions, and regulation of autophagy and apoptosis, providing important pathways that can be investigated more closely [47]. Another integrative approach is expression quantitative trait loci (eQTLs) analysis that leverages gene loci identified from GWAS and integrate these with gene expression data to identify differential gene expression levels to try and uncover the 'molecular phenotype' that lead to these variations in exercise response $[82,83]$. Willems et al. identified the rs6565586 SNP in ACTG1 as a strong candidate gene in inter-individual variability in the resistance-related phenotype (hand grip strength) and correlated this with a lower expression of mRNA in skeletal muscle. ACTG1 encodes Actin Gamma 1 and is involved in the structure and function of skeletal muscle fibres. Interestingly, in a knock out mouse model, animals displayed overt muscle weakness [84]. This type of analysis presented an ideal candidate gene to begin understanding the molecular mechanisms in human skeletal muscle.

To establish causality of genetic variants in aerobic and resistance trainability the field needs to move forward beyond association analysis. The type of follow-up experiment will depend on the location of SNP within the gene. For SNPs within coding regions ideally experiments are performed to study the effect of the SNP has on protein structure and function. For SNPs within in non-coding regions it more difficult to determine as they may not directly affect a gene but alter/regulate transcription factors and mediate alterations in genes this way [77]. However, with the introduction of the large epigenetic database ENCODE (Encyclopaedia of DNA elements) we can now identify the transcription factor association, chromatin structure and histone modification of target genes [85] and more recently enhancers providing candidate gene targets for follow up analysis [86]. With the discovery of CRISPR Cas-9 genome-editing tool in 2012 [87], this has paved the way for establishing causality of SNPs and the functional effects of them. This has been used to great effect for establishing causal genes implicated in insulin resistance whereby they were able to determine the casual effect of 12 candidate genes that had previously been identified in a GWAS [88]. To date no experiments have been conducted using this gene-editing tool to establish the function and causality of candidate genes of trainability beyond association analysis.

There is still much work to do before personalised exercise prescription (both in a clinical and elite athlete setting) can be based on an individual's genetics. However, there are concerted efforts taking place to make this possible such as the Athlome Project Consortium and the Gene SMART (Skeletal Muscle Response to Training), recently launched with the aim of uncovering the genetic variation underlying athletic performance, adaptation to exercise training, and exercise-related musculoskeletal injuries [89, 90]. These, and other initiatives will allow for population-based approach to understand the role of genes and environmental factors contributing to the complex exercise response phenotype [91]. 
This review summarised robust genetic variants that have been associated with aerobic and resistance trainability. To date, there is very little literature ascribed to understanding the interplay between genes and environmental factors and the development of physiological traits. Therefore, much work remains to identify causal variants and functional relevance of genes associated with aerobic and resistance trainability.

\section{Conflict of interest}

The authors declare that they have no conflict of interest.

\section{References}

[1] Bishop DJ, Granata C, Eynon N. Can we optimise the exercise training prescription to maximise improvements in mitochondria function and content? Biochim Biophys Acta 2014; 1840 1266-1275

[2] Wilson MG, Ellison GM, Cable NT. Basic science behind the cardiovascular benefits of exercise. Br J Sports Med 2016; 50: 93-99

[3] Silverman MN, Deuster PA. Biological mechanisms underlying the role of physical fitness in health and resilience. Interface Focus 2014; 4: 20140040

[4] Bouchard C. Human adaptability may have a genetic basis. In: Landry (Ed). Health Risk Estimation, Risk Reduction and Health Promotion. Proceedings of the 18 th annual meeting of the Society of Prospective Medicine Ottawa: Canadian Public Health Association; 1983: 463-476

[5] Bouchard C. DNA sequence variations contribute to variability in fitness and trainability. Med Sci Sports Exerc 2019; 51: 1781-1785

[6] Voisin S, Jacques M, Lucia A et al. Statistical considerations for exercise protocols aimed at measuring trainability. Exerc Sport Sci Rev 2019; 47: 37-45

[7] Mann TN, Lamberts RP, Lambert MI. High responders and low responders: Factors associated with individual variation in response to standardized training. Sports Med 2014; 44: 1113-1124

[8] Kohrt WM, Malley MT, Coggan AR et al. Effects of gender, age, and fitness level on response of VO2max to training in 60-71 yr olds. J Appl Physiol (1985) 1991; 71: 2004-2011

[9] Scharhag-Rosenberger F, Walitzek S, Kindermann W et al. Differences in adaptations to 1 year of aerobic endurance training: Individual patterns of nonresponse. Scand J Med Sci Sports 2012; 22: 113-118

[10] McPhee JS, Williams AG, Degens $\mathrm{H}$ et al. Inter-individual variability in adaptation of the leg muscles following a standardised endurance training programme in young women. Eur J Appl Physiol 2010; 109: $1111-1118$

[11] McPhee JS, Williams AG, Perez-Schindler J et al. Variability in the magnitude of response of metabolic enzymes reveals patterns of co-ordinated expression following endurance training in women. Exp Physiol 2011; 96: 699-707

[12] Ahtiainen JP, Walker S, Peltonen $\mathrm{H}$ et al. Heterogeneity in resistance training-induced muscle strength and mass responses in men and women of different ages. Age (Dordr) 2016; 38: 10

[13] Jones N, Kiely J, Suraci B et al. A genetic-based algorithm for personalized resistance training. Biol Sport 2016; 33: 117-126

[14] Zempo H, Miyamoto-Mikami E, Kikuchi $\mathrm{N}$ et al. Heritability estimates of muscle strength-related phenotypes: A systematic review and meta-analysis. Scand J Med Sci Sports 2017; 27: 1537-1546
[15] Bouchard C, Leon AS, Rao DC et al. The HERITAGE family study. Aims, design, and measurement protocol. Med Sci Sports Exerc 1995; 27: 721-729

[16] Bouchard C, Daw EW, Rice T et al. Familial resemblance for $\mathrm{VO}_{2}$ max in the sedentary state: the HERITAGE family study. Med Sci Sports Exerc 1998; 30: 252-258

[17] Bouchard C, An P, Rice T et al. Familial aggregation of VO(2max) response to exercise training: Results from the HERITAGE Family Study. J Appl Physiol (1985) 1999; 87: 1003-1008

[18] Bouchard C, Rankinen T, Timmons JA. Genomics and genetics in the biology of adaptation to exercise, in comprehensive physiology. Compr Physiol 2011; 1(3): 1603-1648

[19] Ahmetov I, Kulemin N, Popov D et al. Genome-wide association study identifies three novel genetic markers associated with elite endurance performance. Biol Sport 2015; 32: 3-9

[20] Leońska-Duniec A, Jastrzębski Z, Jażdżewska A et al. Leptin and leptin receptor genes are associated with obesity-related traits changes in response to aerobic training program. J Strength Cond Res 2018; 32: 1036-1044

[21] Miotto PM, Holloway GP. Exercise-induced reductions in mitochondrial ADP sensitivity contribute to the induction of gene expression and mitochondrial biogenesis through enhanced mitochondrial H2O2 emission. Mitochondrion 2019; 46: 116-122

[22] McKenzie JA, Witkowski S, Ludlow AT et al. AKT1 G205T genotype influences obesity-related metabolic phenotypes and their responses to aerobic exercise training in older Caucasians. Exp Physiol 2011; 96: 338-347

[23] Bouchard C, Sarzynski MA, Rice TK et al. Genomic predictors of the maximal $\mathrm{O}(2)$ uptake response to standardized exercise training programs. J Appl Physiol (1985) 2011; 110: 1160-1170

[24] Soci UPR, Melo SFS, Gomes JLP et al. Exercise training and epigenetic regulation: multilevel modification and regulation of gene expression. Adv Exp Med Biol 2017; 1000: 281-322

[25] Cagnin S, Chemello F, Ahmetov II. Genes and response to aerobic training. In: Barh D and Ahmetov II, (Eds). Sports, Exercise, and Nutritional Genomics: Current Status and Future DirectionsElsevier Academic Press; London, UK: 2019: 169-188

[26] Huffman JE. Examining the current standards for genetic discovery and replication in the era of mega-biobanks. Nat Commun 2018; 9: 5054

[27] Chanock SJ, Manolio T, Boehnke M et al. Replicating genotype-phenotype associations. Nature 2007; 447: 655-660

[28] Harriss D], MacSween A, Atkinson G. Ethical standards in sport and exercise science research: 2020 update. Int J Sports Med 2019; 40: 813-817

[29] Tam V, Patel N, Turcotte M et al. Benefits and limitations of genome-wide association studies. Nat Rev Genet 2019; 20: 467-484

[30] Zadro JR, Shirley D, Andrade TB et al. the beneficial effects of physical activity: Is It Down to Your Genes? A systematic review and meta-analysis of twin and family studies. Sports Med Open 2017; 3: 4

[31] Ghosh S, Vivar JC, Sarzynski MA et al. Integrative pathway analysis of a genome-wide association study of $\mathrm{VO} 2 \mathrm{max}$ response to exercise training. J Appl Physiol (1985) 2013; 115: 1343-1359

[32] Petr M, Stastny P, Zajac A et al. The role of peroxisome proliferatoractivated receptors and their transcriptional coactivators gene variations in human trainability: A systematic review. Int J Mol Sci 2018; 19: 1472

[33] Franks PW, Barroso I, Luan J et al. PGC-1alpha genotype modifies the association of volitional energy expenditure with $\mathrm{VO}_{2}$ max. Med Sci Sports Exerc 2003; 35: 1998-2004 
[34] Wang YX, Zhang CL, Yu RT et al. Regulation of muscle fiber type and running endurance by PPARdelta. PLoS Biol 2004; 2: e294

[35] Hautala AJ, Leon AS, Skinner JS et al. Peroxisome proliferator-activated receptor- $\delta$ polymorphisms are associated with physical performance and plasma lipids: The HERITAGE Family Study. Am J Physiol Heart Circ Physiol 2007; 292: H2498-H2505

[36] Skogsberg J, Kannisto K, Cassel TN et al. Evidence that peroxisome proliferator-activated receptor delta influences cholesterol metabolism in men. Arterioscler Thromb Vasc Biol 2003; 23: 637-643

[37] Karpe F, Ehrenborg EE. PPARdelta in humans: Genetic and pharmacological evidence for a significant metabolic function. Curr Opin Lipidol 2009; 20: 333-336

[38] Stefan N, Thamer C, Staiger $\mathrm{H}$ et al. Genetic variations in PPARD and PPARGC1A determine mitochondrial function and change in aerobic physical fitness and insulin sensitivity during lifestyle intervention. J Clin Endocrinol Metab 2007; 92: 1827-1833

[39] Franks PW, Christophi CA, Jablonski KA et al. Common variation at PPARGC $1 \mathrm{~A} / \mathrm{B}$ and change in body composition and metabolic traits following preventive interventions: The Diabetes Prevention Program. Diabetologia 2014; 57: 485-490

[40] Ring-Dimitriou S, Kedenko L, Kedenko I et al. Does Genetic Variation in PPARGC1A Affect Exercise-Induced Changes in Ventilatory Thresholds and Metabolic Syndrome? J Exerc Physiol Online 2014; 17: $1-18$

[41] Steinbacher P, Feichtinger RG, Kedenko $L$ et al. The single nucleotide polymorphism Gly482Ser in the PGC-1alpha gene impairs exerciseinduced slow-twitch muscle fibre transformation in humans. PLoS One 2015; 10: e0123881

[42] Pickering C, Kiely J, Suraci B et al. The magnitude of Yo-Yo test improvements following an aerobic training intervention are associated with total genotype score. PLoS One 2018; 13: e0207597

[43] $\mathrm{He} Z$, Hu Y, Feng L et al. Is there an association between PPARGC1A genotypes and endurance capacity in Chinese men? Scand J Med Sci Sports 2008; 18: 195-204

[44] He ZH, Hu Y, Li Y-C et al. PGC-related gene variants and elite endurance athletic status in a Chinese cohort: a functional study. Scand J Med Sci Sports 2015; 25: 184-195

[45] Lin J, Wu H, Tarr PT et al. Transcriptional co-activator PGC-1 alpha drives the formation of slow-twitch muscle fibres. Nature 2002; 418: 797-801

[46] Huang T, Shu Y, Cai YD. Genetic differences among ethnic groups. BMC Genomics 2015; 16: 1093

[47] Sarzynski MA, Ghosh S, Bouchard C. Genomic and transcriptomic predictors of response levels to endurance exercise training. J Physiol 2017; 595: 2931-2939

[48] Lobo S, Wiczer BM, Bernlohr DA. Functional analysis of long-chain acyl-CoA synthetase 1 in 3T3-L1 adipocytes. J Biol Chem 2009; 284: 18347-18356

[49] Timmons JA, Knudsen S, Rankinen T et al. Using molecular classification to predict gains in maximal aerobic capacity following endurance exercise training in humans. J Appl Physiol (1985) 2010; 108: $1487-1496$

[50] Del Coso J, Hiam D, Houweling P et al. More than a 'speed gene': ACTN3 R577X genotype, trainability, muscle damage, and the risk for injuries. Eur J Appl Physiol 2019; 119: 49-60

[51] Sparks MA, Crowley SD, Gurley SB et al. Classical Renin-Angiotensin system in kidney physiology. Compr Physiol 2014; 4: 1201-1228

[52] Rigat B, Hubert C, Alhenc-Gelas F et al. An insertion/deletion polymorphism in the angiotensin I-converting enzyme gene accounting for half the variance of serum enzyme levels. J Clin Invest 1990; 86: 1343-1346
[53] Alves CR, Fernandes T, Lemos JR Jr et al. Aerobic exercise training differentially affects ACE C- and N-domain activities in humans: Interactions with ACE I/D polymorphism and association with vascular reactivity. J Renin Angiotensin Aldosterone Syst 2018; 19: 1470320318761725

[54] Ma F, Yang Y, Li X et al. The association of sport performance with ACE and ACTN3 genetic polymorphisms: A systematic review and meta-analysis. PLoS One 2013; 8: e54685

[55] Charbonneau DE, Hanson ED, Ludlow AT et al. ACE genotype and the muscle hypertrophic and strength responses to strength training. Med Sci Sports Exerc 2008; 40: 677-683

[56] Folland J, Leach B, Little T et al. Angiotensin-converting enzyme genotype affects the response of human skeletal muscle to functional overload. Exp Physiol 2000; 85: 575-579

[57] Giaccaglia V, Nicklas B, Kritchevsky S et al. Interaction between angiotensin converting enzyme insertion/deletion genotype and exercise training on knee extensor strength in older individuals. Int J Sports Med 2008; 29: 40-44

[58] Pereira A, Costa AM, Izquierdo M et al. ACE I/D and ACTN3 R/X polymorphisms as potential factors in modulating exercise-related phenotypes in older women in response to a muscle power training stimuli. Age (Dordr) 2013; 35: 1949-1959

[59] He L, Zhang X, Lv Y et al. Effects of 8 weeks of moderate-intensity resistance training on muscle changes in postmenopausal women with different angiotensin-converting enzyme insertion/deletion polymorphisms of interest. Menopause 2019; 26: 899-905

[60] Pescatello LS, Kostek MA, Gordish-Dressman H et al. ACE ID genotype and the muscle strength and size response to unilateral resistance training. Med Sci Sports Exerc 2006; 38: 1074-1081

[61] Erskine RM, Williams AG, Jones DA et al. The individual and combined influence of ACE and ACTN3 genotypes on muscle phenotypes before and after strength training. Scand J Med Sci Sports 2014; 24: 642-648

[62] Ginevičiene V, Pranculis A, Jakaitienè A et al. Genetic variation of the human ACE and ACTN3 genes and their association with functional muscle properties in Lithuanian elite athletes. Medicina (Kaunas) 2011; 47: 284-290

[63] Thomis MA, Huygens W, Heuninckx S et al. Exploration of myostatin polymorphisms and the angiotensin-converting enzyme insertion/ deletion genotype in responses of human muscle to strength training. Eur J Appl Physiol 2004; 92: 267-274

[64] Pescatello LS, Devaney JM, Hubal MJ et al. Highlights from the functional single nucleotide polymorphisms associated with human muscle size and strength or FAMuSS study. Biomed Res Int 2013; 2013: 643575

[65] Ash GI, Kostek MA, Lee H et al. Glucocorticoid receptor (NR3C1) variants associate with the muscle strength and size response to resistance training. PLoS One 2016; 11: e0148112

[66] Clarkson PM, Devaney JM, Gordish-Dressman H et al. ACTN3 genotype is associated with increases in muscle strength in response to resistance training in women. J Appl Physiol (1985) 2005; 99: 154-163

[67] Harmon BT, Orkunoglu-Suer EF, Adham K et al. CCL2 and CCR2 variants are associated with skeletal muscle strength and change in strength with resistance training. J Appl Physiol (1985) 2010; 109: $1779-1785$

[68] Sprouse C, Gordish-Dressman H, Orkunoglu-Suer EF et al. SLC30A8 nonsynonymous variant is associated with recovery following exercise and skeletal muscle size and strength. Diabetes 2014; 63: 363-368 
[69] Walsh S, Haddad C], Kostek MA et al. Leptin and leptin receptor genetic variants associate with habitual physical activity and the arm body composition response to resistance training. Gene 2012; 510: $66-70$

[70] Pistilli EE, Devaney JM, Gordish-Dressman H et al. Interleukin-15 and interleukin-15R alpha SNPs and associations with muscle, bone, and predictors of the metabolic syndrome. Cytokine 2008; 43: 45-53

[71] Riechman SE, Balasekaran G, Roth SM et al. Association of interleukin-15 protein and interleukin- 15 receptor genetic variation with resistance exercise training responses. J Appl Physiol (1985) 2004; 97: 2214-2219

[72] Loro E, Bisetto S, Khurana TS. Mitochondrial ultrastructural adaptations in fast muscles of mice lacking IL15RA. J Cell Sci 2018; 131

[73] Walsh S, Kelsey BK, Angelopoulos T] et al. CNTF 1357 G -> A polymorphism and the muscle strength response to resistance training. J Appl Physiol (1985) 2009; 107: 1235-1240

[74] Hong AR, Hong SM, Shin YA. Effects of resistance training on muscle strength, endurance, and motor unit according to ciliary neurotrophic factor polymorphism in male college students. J Sports Sci Med 2014; 13: 680-688

[75] Khramtsova EA, Davis LK, Stranger BE. The role of sex in the genomics of human complex traits. Nat Rev Genet 2019; 20: 173-190

[76] Landen S, Voisin S, Craig JM et al. Genetic and epigenetic sex-specific adaptations to endurance exercise. Epigenetics 2019; 14: 523-535

[77] Karczewski KJ, Snyder MP. Integrative omics for health and disease. Nat Rev Genet 2018; 19: 299-310

[78] Sun YV, Hu Y]. Integrative Analysis of Multi-omics Data for Discovery and Functional Studies of Complex Human Diseases. Adv Genet 2016; 93: 147-190

[79] Battle A, Khan Z, Wang SH et al. Genomic variation. Impact of regulatory variation from RNA to protein. Science 2015; 347 : 664-667

[80] Veyrieras JB, Kudaravalli S, Kim SY et al. High-resolution mapping of expression-QTLs yields insight into human gene regulation. PLoS Genet 2008; 4: e1000214

[81] Taylor DL, Jackson AU, Narisu N et al. Integrative analysis of gene expression, DNA methylation, physiological traits, and genetic variation in human skeletal muscle. Proc Natl Acad Sci USA 2019; 116: $10883-10888$

[82] Nicolae DL, Gamazon E, Zhang W et al. Trait-associated SNPs are more likely to be eQTLs: Annotation to enhance discovery from GWAS. PLoS Genet 2010; 6: e1000888

[83] Keildson S, Fadista J, Ladenvall C et al. Expression of phosphofructokinase in skeletal muscle is influenced by genetic variation and associated with insulin sensitivity. Diabetes 2014; 63: 1154-1165

[84] Sonnemann KJ, Fitzsimons DP, Patel JR et al. Cytoplasmic gammaactin is not required for skeletal muscle development but its absence leads to a progressive myopathy. Dev Cell 2006; 11: 387-397

[85] Dunham I, Kundaje A, Aldred SF et al. An integrated encyclopedia of DNA elements in the human genome. Nature 2012; 489: 57-74

[86] Fishilevich S, Nudel R, Rappaport N et al. GeneHancer: Genome-wide integration of enhancers and target genes in GeneCards. Database (Oxford) 2017; 2017: bax028

[87] Jinek M, Chylinski K, Fonfara I et al. A Programmable Dual-RNA-Guided DNA Endonuclease in Adaptive Bacterial Immunity. Science 2012; 337: 816-821

[88] Chen Z, Yu H, Shi X et al. Functional screening of candidate causal genes for insulin resistance in human preadipocytes and adipocytes. Circ Res 2020; 126: 330-346
[89] Wang G, Tanaka M, Eynon N et al. The future of genomic research in athletic performance and adaptation to training. Med Sport Sci 2016; 61: 55-67

[90] Yan X, Eynon N, Papadimitriou ID et al. The gene SMART study: Method, study design, and preliminary findings. BMC Genomics 2017; 18 (Suppl 8): 821

[91] Baliga NS, Björkegren JLM, Boeke JD et al. The State of Systems Genetics in 2017. Cell Syst 2017; 4: 7-15

[92] Alves GB, Oliveira EM, Alves CR et al. Influence of angiotensinogen and angiotensin-converting enzyme polymorphisms on cardiac hypertrophy and improvement on maximal aerobic capacity caused by exercise training. Eur J Cardiovasc Prev Rehabil 2009; 16: 487-492

[93] Dionne FT, Turcotte L, Thibault MC et al. Mitochondrial DNA sequence polymorphism, $\mathrm{VO}_{2}$ max, and response to endurance training. Med Sci Sports Exerc 1991; 23: 177-185

[94] He Z, Hu Y, Feng L et al. NRF-1 genotypes and endurance exercise capacity in young Chinese men. Br J Sports Med 2008; 42: 361-366

[95] He Z, Hu Y, Feng L et al. Polymorphisms in the HBB gene relate to individual cardiorespiratory adaptation in response to endurance training. Br J Sports Med 2006; 40: 998-1002

[96] He Z, Hu Y, Feng L et al. NRF2 genotype improves endurance capacity in response to training. Int J Sports Med 2007; 28: 717-721

[97] He Z-H, Hu Y, Wang H-Y et al. Are calcineurin genes associated with endurance phenotype traits? Eur J Appl Physiol 2010; 109: 359-369

[98] He ZH, Hu Y, Li Y-C et al. Polymorphisms in the calcineurin genes are associated with the training responsiveness of cardiac phenotypes in Chinese young adults. Eur J Appl Physiol 2010; 110: 761-767

[99] Leon AS, Togashi K, Rankinen T et al. Association of apolipoprotein E polymorphism with blood lipids and maximal oxygen uptake in the sedentary state and after exercise training in the HERITAGE family study. Metabolism 2004; 53: 108-116

[100] McPhee JS, Perez-Schindler ], Degens H et al. HIF1A P582S gene association with endurance training responses in young women. Eur ] Appl Physiol 2011; 111: 2339-2347

[101] Prior SJ, Hagberg JM, Phares DA et al. Sequence variation in hypoxia-inducible factor 1 (HIF1A): Association with maximal oxygen consumption. Physiol Genomics 2003; 15: 20-26

[102] Prior SJ, Hagberg JM, Paton CM et al. DNA sequence variation in the promoter region of the VEGF gene impacts VEGF gene expression and maximal oxygen consumption. Am J Physiol Heart Circ Physiol 2006; 290: H1848-H1855

[103] Rankinen T, Pérusse L, Borecki I et al. The Na K ATPase 2 gene and trainabiity of cardiorespiratory endurance: The HERITAGE Family Study. J Appl Physiol (1985) 2000; 88: 346-351

[104] Rankinen T, Gagnon J, Pérusse L et al. AGT M235T and ACE ID polymorphisms and exercise blood pressure in the HERITAGE Family Study. Am J Physiol Heart Circ Physiol 2000; 279: 368-374

[105] Rico-Sanz J, Rankinen T, Joanisse DR et al. Associations between cardiorespiratory responses to exercise and the C34T AMPD1 gene polymorphism in the HERITAGE Family study. Physiol Genomics 2003; 14: 161-166

[106] Rivera MA, Dionne FT, Simoneau JS et al. Muscle-specific creatine kinase gene polymorphism and VO2max in the HERITAGE Family Study. Med Sci Sports Exerc 1997; 29: 1311-1317

[107] Sonna LA, Sharp MA, Knapik J] et al. Angiotensin-converting enzyme genotype and physical performance during US Army basic training. J Appl Physiol (1985) 2001; 91: 1355-1363

[108] Yoo J, Kim B-H, Kim S-H et al. Genetic polymorphisms to predict gains in maximal $\mathrm{O} 2$ uptake and knee peak torque after a high intensity training program in humans. Eur J Appl Physiol 2016; 116: 947-957 
[109] Yu B, Chen W, Wang R et al. Association of apolipoprotein E polymorphism with maximal oxygen uptake after exercise training: a study of Chinese young adult. Lipids Health Dis 2014; 13: 40

[110] Zarebska A, Jastrzebski Z, Kaczmarczyk M et al. The Gstp1 C.313a > G polymorphism modulates the cardiorespiratory response to aerobic training. Biol Sport 2014; 31: 261-266

[111] Zhou DQ, Hu Y, Liu G et al. Muscle-specific creatine kinase gene polymorphism and running economy responses to an 18-week 5000-m training programme. Br J Sports Med 2006; 40: 988-991

[112] Delmonico MJ, Kostek MC, Doldo NA et al. Alpha-actinin-3 (ACTN3) R577X polymorphism influences knee extensor peak power response to strength training in older men and women. J Gerontol A Biol Sci Med Sci 2007; 62: 206-212

[113] Erskine RM, Williams AG, Jones DA et al. Do PTK2 gene polymorphisms contribute to the interindividual variability in muscle strength and the response to resistance training? A preliminary report. J Appl Physiol (1985) 2012; 112: 1329-1334
[114] Jamshidi Y, Montgomery HE, Hense H-W et al. Peroxisome proliferator-activated receptor alpha gene regulates left ventricular growth in response to exercise and hypertension. Circulation 2002; 105: 950-955

[115] Keogh JW, Palmer BR, Taylor D et al. ACE and UCP2 gene polymorphisms and their association with baseline and exercise-related changes in the functional performance of older adults. Peer] 2015; 3: e980

[116] Kostek MC, Delmonico MJ, Reichel JB et al. Muscle strength response to strength training is influenced by insulin-like growth factor 1 genotype in older adults. J Appl Physiol (1985) 2005; 98: 2147-2154

[117] Li X, Wang S-J, Tan SC et al. The A55T and K153R polymorphisms of MSTN gene are associated with the strength training-induced muscle hypertrophy among Han Chinese men. J Sports Sci 2014; 32: 883-891 